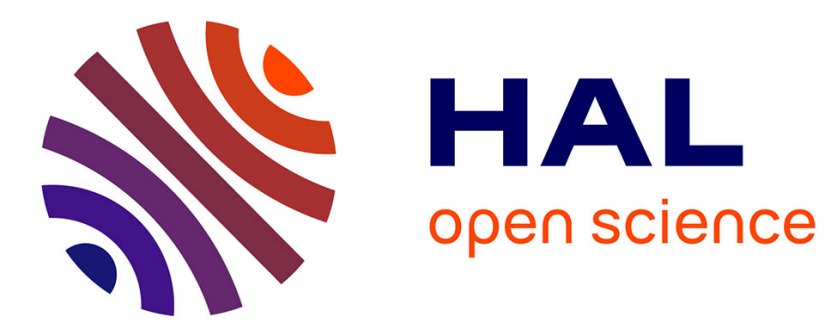

\title{
Normalized Nash Equilibrium for Power Allocation in Cognitive Radio Networks
}

\author{
Arnob Ghosh, Laura Cottatellucci, Eitan Altman
}

\section{To cite this version:}

Arnob Ghosh, Laura Cottatellucci, Eitan Altman. Normalized Nash Equilibrium for Power Allocation in Cognitive Radio Networks. IEEE Transactions on Cognitive Communications and Networking, 2015, 1 (1), pp.86-99. 10.1109/TCCN.2015.2496578 . hal-01234824

\section{HAL Id: hal-01234824 \\ https://hal.inria.fr/hal-01234824}

Submitted on 1 Dec 2015

HAL is a multi-disciplinary open access archive for the deposit and dissemination of scientific research documents, whether they are published or not. The documents may come from teaching and research institutions in France or abroad, or from public or private research centers.
L'archive ouverte pluridisciplinaire HAL, est destinée au dépôt et à la diffusion de documents scientifiques de niveau recherche, publiés ou non, émanant des établissements d'enseignement et de recherche français ou étrangers, des laboratoires publics ou privés. 


\title{
Normalized Nash Equilibrium for Power Allocation in Cognitive Radio Networks
}

\author{
Arnob Ghosh*, Laura Cottatellucci ${ }^{\dagger}$, and Eitan Altman ${ }^{\ddagger}$
}

\begin{abstract}
We consider a cognitive radio system consisting of secondary networks and several primary user-terminals (primary-UTs). In a secondary network a secondary-base station (secondary-BS) transmits to a secondary-user terminal (secondary-UT) with certain power. Secondary-BSs are constrained to allocate transmitting powers such that the total interference at each primary-UT is below a given threshold. We formulate the power allocation problem as a concave non cooperative game with secondary-BSs as players and multiple primary-UTs enforcing coupled constraints. The equilibrium selection is based on the concept of normalized Nash equilibrium (NNE). When the interference at a secondary-UT from adjacent secondary-BSs is negligible, the $\mathrm{NNE}$ is shown to be unique for any strictly concave nondecreasing utility. The NNE is also shown to be the solution of a concave potential game. We propose a distributed algorithm which converges to the unique NNE. When the interference at a secondary-UT from adjacent secondary-BSs is not negligible, an NNE may not be unique and the computation of the NNE has exponential complexity. To avoid these drawbacks, we introduce the concept of weakly normalized Nash equilibrium (WNNE) which keeps the most of NNEs' interesting properties but, in contrast to the latter, the WNNE can be determined with low complexity. We show the usefulness of the WNNE concept for the relevant case of Shannon capacity.
\end{abstract}

\section{INTRODUCTION}

A traditional static spectrum access leads to spectrum underutilization. Cognitive radio can enhance the spectrum utilization if primary network providers (license spectrum holders) allow secondary users (unlicensed users) to access the licensed spectrum provided that the primary users (subscribers of the primary network providers) are protected from the interference of secondary users [2]. Without proper policies for power and frequency band allocation, the transmission rates at primaryUTs' would degrade significantly and thus, a primary network provider would not allow secondary users to access the spectrum. Therefore, in a secondary network a secondary-BS must select its transmit power using cognitive radio technology such that the total interference from secondary-BSs at each primaryUT is below an acceptable threshold.

In practice, each secondary-BS is an independent entity and selects its transmission power level in order to maximize only its own utility ${ }^{1}$. Thus, a non cooperative game theoretic setting

Part of this paper was presented in WiOpt'15 [1].

* The author is in Electrical and Systems Engineering Dept., University of Pennsylvania, USA; E-mail Id: arnob@seas.upenn.edu

$\dagger$ The author is with Mobile Communication Dept., EURECOM, Sophia Antipolis, France; E-mail Id:laura.cottatellucci@eurecom.fr

$\ddagger$ The author is with INRIA Sophia Antipolis, 10 route des Lucioles, Sophia Antipolis, France; E-mail id:eitan.altman@inria.fr

${ }^{1}$ Secondary-BSs are either installed by different secondary service providers (which are unlicensed local service providers) or by end-users. Hence, they are likely to independently maximize their own utilities. is a preferable model in this context. A distributed algorithm is also preferable to obtain an equilibrium in order to keep the exchange of information among the secondary-BSs limited. Since the secondary-BSs are not operated by the primaryBS owners and primary-UTs are oblivious of the number of active secondary-BSs, the exchange of information between primary- and secondary-BSs to obtain an equilibrium should also be limited. To summarize, our goal is to obtain an equilibrium power allocation strategy profile using a distributed approach based on a non cooperative game theoretic setting with secondary-BSs as players and with a minimal exchange of information among the secondary-BSs. Additionally, the primary-UTs should also be oblivious of the secondary-BSs.

Our Contributions: We consider a setting with multiple secondary-BSs and multiple primary-UTs. Each secondary-BS serves only one secondary-UT. We formulate the power allocation problem among secondary-BSs as a coupled constrained concave game [3] with secondary-BSs as players. Hence, the strategy of a player as well as its utility also depends on the strategy of other players. There are multiple Nash equilibriums (NEs) in the coupled constrained concave game in general. In order to design distributed algorithms converging to a well defined and unique equilibrium we resort to the concept of NNE introduced by Rosen in [3].

In Section II we present two scenarios, in the first one the interference at a secondary-UT from other secondary-BSs, i.e., the inter-secondary-network interference is negligible. This scenario is likely to arise when the secondary-BSs have small coverage area and the number of secondary networks are small. In the second scenario, the inter secondary network interference is non negligible and the utilities of secondaryBSs directly depend on the policies selected by the other secondary-BSs. This situation is likely to arise when the coverage of secondary-BS is large and/or the number of secondary networks are large. The analysis of the former scenario is ancillary to the analysis of the latter one and motivates the introduction of the concept of WNNE to extend the appealing properties of the NNE in the former scenario to systems with inter-secondary network interference.

In Section VI, we show that the computation of an NNE reduces to solving a convex optimization problem when the game admits a concave potential function [4]. We propose a distributed algorithm which converges to the NNE when the game admits a strictly concave potential function. In the algorithm (Algorithm DIST, Section VI-B) the secondary-BSs do not need to exchange information among them and the primary-UTs only need to track the total interference. PrimaryUTs select prices for the total interference caused by the secondary-BSs. This mechanism also provides an incentive 
to primary-UTs to cooperate in the convergence process of the algorithm. We show that in the setting with negligible interference among secondary-UTs, the NNE is always unique and the game admits a strictly concave potential function. Thus, the algorithm DIST can be used in this setting to obtain the unique NNE.

In the setting with inter-secondary-network interference, the NNE may not be unique and the game does not necessarily admit a potential function. Nevertheless, we identify a class of utility functions which admits unique NNEs and strictly concave potential functions even in presence of co-channel interference among secondary-BSs. Thus, algorithm DIST can be used to obtain the unique NNE. We introduce the concept of Weakly Normalized Nash equilibrium (WNNE) as an equilibrium selection concept when it is difficult to compute a NNE in presence of inter-secondary-network interference. In Section VI-D we illustrate the significance of the WNNE by analyzing a specific game with a function that provides an achievable rate, commonly referred to as Shannon function, as a utility function and inter-secondary network interference. This game admits a unique NNE only under certain conditions depending on the parameters of the channels and it does not admit a potential function. The standard techniques to compute NNE in this game have exponential complexity. We provide an algorithm whose complexity only scales linearly with the number of secondary-BSs. The implementation of this algorithm requires that a secondary-BS knows all the channel coefficients from each secondary-BS to all secondaryUTs. On the contrary, the WNNE can be obtained with lower complexity using the distributed algorithm DIST. When algorithm DIST is applied, each secondary-BS only needs to know the coefficients of the local channels from the BS itself and all the primary-UTs. Moreover, WNNEs can be obtained for all possible realizations of the channel parameters. Finally, we numerically evaluate various properties of NNE and WNNE solution for some well known utility functions in Section VIII.

Related Literature: Game theoretic approaches have been widely applied to wireless communication problems (see e.g. [5]). However, only those works which are related to resource allocation in heterogeneous or cognitive radio network are of particular interest to us. Algorithms for power allocation at the secondary-UTs relying on the cooperation of primary-UTs have been studied in [6], [7]. In [8], [9], the authors also considered an active participation of primary-UTs in order to allocate the transmission power among secondary-UTs. Power allocation in cognitive radio using Stackelberg game is studied [10], [11] with primary-UTs as leaders and secondary-BSs as followers. Power allocation for heterogeneous networks (HetNets) using Stackelberg game is studied in [12], [13]. The setting of HetNet is analogous to the setting considered in this article with macro- and femto-cells playing the role of primary and secondary networks, respectively. In contrast to the above mentioned works, in the game theoretical framework proposed in this article, primary-UTs are oblivious of the number of secondary-BSs. Primary-UTs are almost passive entities that only select prices depending on the total interference: no knowledge of each secondary-BS's utility or the channel state information is required. Thus, our model is readily scalable compared to previous models. In [14], the authors proposed a Nash bargaining solution as power allocation among secondary users. The solution in [14] implies a cooperation among secondary-BSs whereas we consider a non cooperative game theoretic setting where each secondary-BS only maximizes its own utility. In [15], the authors obtained an equilibrium power allocation strategy using a non cooperative game theoretic distributed algorithm. However, no interference constraints are enforced at the primary-UTs, i.e., the total interference at each primary-UT is not constrained to be below a certain threshold.

Optimal power allocation in HetNet with interference mitigation techniques is also studied using evolutionary game theoretic approaches [16], [17]. Both these papers assume an identical discrete finite strategy space for each player whereas the strategy space of each player in our setting is continuous, uncountably infinite and the strategy of a player inherently depends on the strategy of other players since we consider a coupled constrained game. Additionally, in [16] and [17] a player must know the average utility of the other players in order to obtain a stable equilibrium and thus, it requires either communications among players [17] or a central controller [16]. In contrast, in our setting a player, i.e., a secondary-BS, does not need to know the utility functions of other secondaryBSs in the distributed algorithm DIST to attain a NNE (or, WNNE). In [18], the authors also studied resource allocation among secondary-UTs using a stochastic learning method. Unlike the DIST algorithm, the method proposed in [18] needs a central controller and coordination among players. Thus, our approach is more practically viable and readily implementable compared to the above mentioned works.

Distributed power allocation in cognitive radio network in a non cooperative game theoretic setting has also been studied in [19]-[21]. Our approach differs from those works in the following aspects. Previous works focused on the characteristics of the equilibriums for very specific utility functions. In contrast, we characterize the uniqueness of the NNE for a wide class of utility functions. Moreover, for the cases when it is difficult to compute the NNE, we introduce the WNNE as an equilibrium selection approach since it retains most of the favorable properties of NNE. Thus, our equilibrium selection methods can be applied to more generalized and challenging settings. Additionally, in the above mentioned works, secondary-BSs need to exchange information among them for the distributed algorithm to converge. In the present work, algorithm DIST, which provides NNE or WNNE, does not require exchange of information among secondary-BSs. Finally, only in [19] the Shannon capacity function is adopted as a utility function. The algorithm proposed in [19] to compute an NE scales exponentially with the number of secondaryBSs. In contrast, for the Shannon capacity function in presence of inter-secondary-network interference, we identify the conditions under which an NNE is unique and we propose an algorithm to obtain the unique NNE which scales only linearly with the number of secondary-BSs.

NNEs have been studied for general coupled constrained games in [22] (see references there in). However, this work did not consider NNEs in cognitive radio settings. Additionally, 


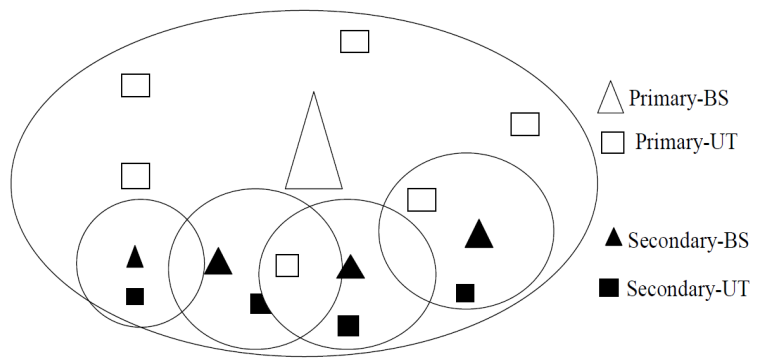

Fig. 1. Primary-BS, primary-UTs, secondary-BSs and secondary-UTs in a region. Circles represent the range of base stations. Primary-BS has higher coverage compared to secondary-BS. Each secondary-BS serves only one secondary-UT. Secondary-UT is placed close to its serving secondary-BS. Secondary-BSs cause interference at a primary-UT as well as the secondaryUTs

[22] did not provide any distributed algorithm to compute an NNE. The closest work to ours is [23] where the NNE is adopted as equilibrium concept for optimal power allocation among femto-BSs in a HetNet. In [23], a single macro-UT with interference free femto-cells is considered. The setting studied there corresponds to a setting with a single primary-UT and negligible inter-secondary-network interference. We relax both these assumptions in this paper. We contribute in this space. The presence of multiple primary-UTs in the system raises a problem of computational complexity in determining the unique NNE: the NNE computation does not boil down to an ordinary water-filling problem as in [23]. By applying standard techniques it turns out that the problem has exponential complexity in the number of primary-UTs and secondary-BSs and we are not aware of the techniques that solve the problem with lower complexity. Along with increasing complexity, the consideration of co-channel interference at secondary-UTs has the effect of destroying the property of uniqueness of NNE always satisfied in the setting considered in [23].

All proofs have been relegated to the section Appendix.

Notation. Vectors and matrices are denoted by bold lower case and bold capital letters, respectively; ${ }^{T}$ denotes the transpose operator; the notation $\mathbf{x} \succeq \mathbf{0}$ stays for componentwise inequality; $\mathbf{I}_{M}$ denotes the $M \times M$ identity matrix and $\mathbf{1}_{M}$ and $\mathbf{0}_{M}$ are the $M$-dimensional column vectors of ones and zeros, respectively. Given the real $x,(x)^{+}=\max (x, 0)$. Additionally, $\mathbf{H}_{K}$ is the sub-matrix of $\mathbf{H}$ containing only the rows and columns in an index set $K$. This notation is immediately extended to vectors. The vector $\mathbf{v}_{-i}$ is obtained from vector $\mathbf{v}$ by suppressing the $i$ th component. The matrix operator $\odot$ denotes the Hadamard or component wise product; $\mathbf{D}=\operatorname{diag}(\mathbf{v})$ maps vector $\mathbf{v}$ onto a diagonal matrix with diagonal component $d_{\ell, \ell}=v_{\ell}$. The set of nonnegative real numbers in denoted by $\mathbb{R}_{+}$.

\section{SySTEM MODEL}

We consider a cognitive radio network consisting of $F$ secondary-BSs and $M$ primary-UTs (Fig. 1) [24]. In each secondary network, a secondary-BS serves a single secondaryUT. Thus, there are $F$ secondary-UTs. We do not make any assumptions regarding the distribution of secondary-BSs, secondary-UTs and secondary-UTs except the fact that each secondary-UT is located close to its secondary-BS since

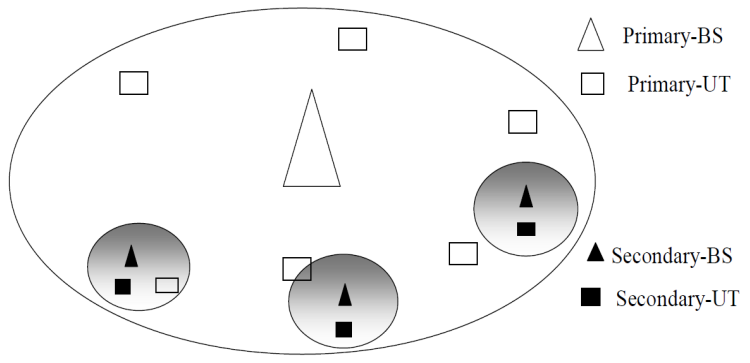

Fig. 2. Primary-BS, primary-UTs, secondary-BSs and secondary-UTs in a region. Circles represent the range of base stations. The range of secondary$\mathrm{BS}$ is smaller compared to Figure 1. The number of secondary networks is lower. In this setting, secondary-BSs do not cause interference at the adjacent secondary-UTs. However, they still cause interference at primary-UTs.

secondary-BS has smaller coverage area. We consider the secondary spectrum access model where secondary networks and a primary network use the same channel for downlink communications. Let $h_{f}$ be the channel gain between the secondary-BS $f$ and its served secondary-UT $f$ and $\widehat{h}_{m}^{f}$ is the channel gain between secondary-BS $f$ and primary-UT $m$. Finally, $\widetilde{h}_{k}^{f}$ is the channel gain between secondary-BS $k$ and secondary-UT $f$, with $f \neq k$. The secondary-BS $f$ transmits with power $p_{f} \geq 0$. For future use, it is convenient to define the following vectors $\mathbf{p}=\left(p_{1}, p_{2}, \ldots, p_{F}\right)^{T}$, $\mathbf{h}=\left(h_{1}, h_{2}, \ldots, h_{F}\right)^{T}, \widehat{\mathbf{h}}_{m}=\left(\widehat{h}_{m}^{1}, \widehat{h}_{m}^{2}, \ldots, \widehat{h}_{m}^{F}\right)^{T}$, with $m=1, \ldots, M, \widehat{\sim}^{f}=\left(\widehat{h}_{1}^{f}, \widehat{h}_{2}^{f}, \ldots, \widehat{h}_{M}^{f}\right)^{T}$, and $\widetilde{\mathbf{h}}^{f}=$ $\left(\widetilde{h}_{1}^{f}, \widetilde{h}_{2}^{f}, \ldots, \widetilde{h}_{f-1}^{f}, \widetilde{h}_{f+1}^{f}, \ldots \widetilde{h}_{F}^{f}\right)^{T}$, with $f=1, \ldots, F$.

The primary network operates in the time division duplexing (TDD) mode i.e. the primary-UTs transmit and receive in the same frequency band. This feature implies that secondary-BS $f$ can estimate $\widehat{h}_{m}^{f}$ by sensing a pilot signal sent by primaryUT $m$ due to the channel reciprocity assumption [25]. Hence, the channel feedback by primary-UTs to secondary-BSs is not required to estimate $\widehat{h}_{m}^{f}$. Also note that secondary-BS $f$ can acquire $\widehat{h}_{m}^{f}$ without communicating with other secondary-BSs or secondary-UTs. The interference from secondary-BSs at a primary-UT $m$ is

$$
I_{m}=\mathbf{p}^{T} \widehat{\mathbf{h}}_{m} \quad m=1, \ldots, M .
$$

The signal to interference and noise ratio (SINR) at secondary-UT $f$ is given by

$$
\gamma_{f}=\frac{p_{f} h_{f}}{\sigma^{2}+\mathbf{p}_{-f}^{T} \widetilde{\mathbf{h}^{f}}}
$$

where $\sigma^{2}$ is the variance of the additive white Gaussian noise that also accounts for interference from primary-BSs. In general $\gamma_{f}$ is a function of $\mathbf{p}$. When it is convenient, we explicitly point out this dependence by writing $\gamma_{f}(\mathbf{p})$, otherwise we omit it and use the short notation $\gamma_{f}$.

Until now, we have discussed a general model where interference at a secondary-UT from other secondary-BSs is not negligible. We also consider the setting depicted in Fig. 2 where the interference at a secondary-UT from other secondary-BSs is negligible. The above setting arises when the number of secondary networks is small and the secondary-BS has small coverage area. In this setting, it is reasonable to assume that $\widetilde{\mathbf{h}}^{f} \cong \mathbf{0}$, and thus, $\gamma_{f}$ reduces to the signal to 
noise ratio (SNR) $\gamma_{f}^{\prime}=\frac{p_{f} h_{f}}{\sigma^{2}}$. We show that in this case, the NNE has favorable properties. In a more general setting such as in Fig. 1, those characteristics of the NNE may not remain valid.

Note that in both of these settings, primary-UTs are ubiquitous and in general are distant from the base station due to the wide coverage area of the primary-BS (consider, for example, a macro cell). Thus, primary-UTs are likely to be present relatively close to secondary-BSs. Additionally, since the distance from the primary-BS to primary-UT is large, the received signal power is very low at a primary-UT compared to the signal power received by the secondary-UTs. As a result, secondary-BSs can generate significant performance degradation to primary-UTs even when the interference at each secondary-UT caused by adjacent secondary-BSs is negligible. Thus, the performance degradation at primary-UT due to the transmission of secondary-BSs can be severe in both the studied settings. In order to keep the quality of the downlink communications in each primary-UT acceptable, total interference from all secondary-BSs must be below an acceptable limit in both of these settings. Specifically, we assume

$$
\mathbf{p}^{T} \widehat{\mathbf{h}}_{m} \leq I_{T} \quad m=1, \ldots, M .
$$

In this article we are interested only in keeping the interference caused by secondary-base stations to each primary-UT below a certain acceptable limit. In fact, in practice, the interference from primary-BSs to primary-UTs is efficiently controlled by proper beamforming design and coordinated beamforming. We consider identical thresholds at different primary-UTs in order to keep notations simple. The extension to the general case with different thresholds is straightforward.

Additionally, the transmit powers are constrained to a maximum value $P_{\text {MAX }}$ such that

$$
\mathbf{p} \preceq P_{\mathrm{MAX}} \mathbf{1}_{F} .
$$

\section{Problem Formulation AND SOLUtion CONCEPT}

\section{A. Problem Formulation}

Secondary-BS $f$ selects its transmission power with the objective of maximizing the quality of its communication in downlink. Its communication quality is characterized by $U_{f}\left(\gamma_{f}\right)$, where $U_{f}(\cdot)$ is a concave nondecreasing function.

We formulate the power allocation at secondary-BSs as a non-cooperative game where each secondary-BS aims to maximize its utility $U_{f}\left(\gamma_{f}\right)$ under constraints ${ }^{2}(3)$.

More specifically, we define this non-cooperative game in a strategic form as

$$
\mathcal{G}=\left\{\mathcal{F}, \mathcal{P},\left\{u_{f}(\mathbf{p})\right\}_{f \in \mathcal{F}}\right\}
$$

where the elements of the game are

- Player set: Set of the secondary-BSs $\mathcal{F}=\{1, \ldots, F\}$;

\footnotetext{
${ }^{2}$ Throughout the rest of this paper, to keep notation and equations compact, we do not consider constraints (4). They are orthogonal and can be immediately embedded in the proposed game theoretical framework. The extension of all the results presented here to the case including these additional constraints is straightforward.
}

- Strategy set: $\mathcal{P}=\left\{\mathbf{p} \mid \mathbf{p} \in \mathbb{R}_{+}^{F}\right.$ and $\mathbf{p}^{T} \widehat{\mathbf{h}}_{m} \leq I_{T}, m=$ $1, \ldots M\}$, where $\mathbb{R}_{+}^{F}$ is the product space of $F$ nonnegative real spaces $\mathbb{R}_{+}$.

- Utility set: the functions $u_{f}(\mathbf{p})$ are defined as $u_{f}(\mathbf{p}) \equiv$ $U_{f}\left(\gamma_{f}(\mathbf{p})\right)=U_{f}\left(\frac{p_{f} h_{f}}{\sigma^{2}+\mathbf{p}_{-f}^{T} \widetilde{\mathbf{h}}^{f}}\right), U_{f}(\cdot)$ is a concave nondecreasing function in $\mathbb{R}_{+}$.

We adopt a NE of the non-cooperative game $\mathcal{G}$ as a power allocation policy for the secondary-BSs. More specifically, the power allocation vector $\mathbf{p}^{*}$ is a Nash Equilibrium (NE), i.e., for every $f \in \mathcal{F}$ and $p_{f}$ such that $\left(p_{1}^{*}, \ldots p_{f-1}^{*}, p_{f}, p_{f+1}^{*}, p_{F}^{*}\right) \in \mathcal{P}$

$$
U_{f}\left(\frac{p_{f}^{*} h_{f}}{\sigma^{2}+\mathbf{p}_{-f}^{* T} \widetilde{\mathbf{h}}^{f}}\right) \geq U_{f}\left(\frac{p_{f} h_{f}}{\sigma^{2}+\mathbf{p}_{-f}^{* T} \widetilde{\mathbf{h}}^{f}}\right) .
$$

By observing that each $U_{f}\left(\frac{p_{f} h_{f}}{\sigma^{2}+\mathbf{p}_{-f}^{T} \widetilde{\mathbf{h}}^{f}}\right)$ is continuous in $\mathbb{R}_{+}^{F}$ and concave for $p_{f} \in \mathbb{R}_{+}$and the set $\mathcal{P}$ is convex and closed, we conclude that $\mathcal{G}$ falls in the class of concave games with coupled constraints studied in [3] and a NE exists [3].

\section{B. Normalized Nash Equilibrium}

The strategy set $\mathcal{P}$ is closed, convex, and bounded ${ }^{3}$. Under the further assumption that the functions $U_{f}$, for all $f=\mathcal{F}$, possess continuous first derivatives, we can use the necessary and sufficient KKT conditions for constrained maxima [26] to obtain conditions satisfied by a NE $\mathbf{p}^{*}$. If $\mathbf{p}^{*}$ is a NE in $\mathcal{P}$, then, there exist $F$ vectors $\boldsymbol{\lambda}^{f}=\left(\lambda_{1}^{f}, \lambda_{2}^{f} \ldots, \lambda_{M}^{f}\right)$ with $\boldsymbol{\lambda}^{f} \geq$ $\mathbf{0}$ such that $\mathbf{p}^{*}$ satisfies the following system of equations

$$
\begin{gathered}
\lambda_{m}^{f}\left(\mathbf{p}^{T} \widehat{\mathbf{h}}_{m}-I_{T}\right)=0, \quad m=1, \ldots M \\
\text { and } f=1, \ldots F \\
\frac{\partial U_{f}\left(\gamma_{f}\right)}{\partial p_{f}}-\sum_{m=1}^{M} \lambda_{m}^{f} \frac{\partial}{\partial p_{f}}\left(\mathbf{p}^{T} \widehat{\mathbf{h}}_{m}-I_{T}\right)=0 f=1, \ldots F
\end{gathered}
$$

We can write (8) as

$$
U_{f}^{\prime}\left(\gamma_{f}\right) \frac{\partial \gamma_{f}}{\partial p_{f}}-\boldsymbol{\lambda}^{f T} \widehat{\mathbf{h}}^{f}=0, \quad f=1, \ldots F .
$$

A NE in the coupled game $\mathcal{G}$ is not unique in general. The uniqueness of an NE in a constrained game is guaranteed only for orthogonal constraints, i.e., when the strategy of a player is independent of other players' strategies [3]. Rosen in [3] has introduced the concept of NNE that provides a useful equilibrium selection criterion for coupled constrained games when the strategy of a player poses restrictions on the strategy of other players as in our setting.

The strategy $\mathbf{p}^{*}$ is a normalized Nash equilibrium (NNE) if the KKT conditions in (7) and (9) are satisfied with ${ }^{4} \boldsymbol{\lambda}_{m}=$

\footnotetext{
${ }^{3}$ Boundedness can be immediately verified if each secondary-BS's transmitted signal impinges on at least one of the primary-UT, i.e. there exists no secondary-BS $f$ such that $\widehat{h}_{m}^{f}=0$ for all $m=1, \ldots, M$. If this will not be the case, it is not of a practical interest to include a secondary-BS that does not cause interference on any primary-UT, as player in the game $\mathcal{G}$.

${ }^{4}$ In [3] an equilibrium is an NNE if the KKT conditions are satisfied for some vector $\mathbf{r}>0$ and $\boldsymbol{\lambda}>=0, \lambda_{m}^{f}=\lambda_{m} / r_{f}$. The NNE that we consider is in accordance with the above definition when $\mathbf{r}=\mathbf{1}$. In [3], $\mathbf{r}$ is used to find an NNE for a weighted utility function, i.e., $U_{f}$ is scaled by $r_{f} U_{F}$. In our setting, $\mathbf{r}=\mathbf{1}$ since we do not consider any weighted utility function.
} 
$\left(\lambda_{m}^{1}, \ldots, \lambda_{m}^{F}\right)=\lambda_{m} \mathbf{1}_{F}^{T}$, i.e., the Lagrangian multipliers are identical for all the players for each given constraint enforced by primary-UT $m$. The concept of NNE has several advantages described in the following.

The Lagrangian multiplier $\lambda_{m}^{f}$ can be viewed as the price per unit of interference caused by player $f$ at primary-UT $m$. Thus, as first advantage, a primary-UT does not have to select different prices for different players in a NNE. Additionally, as it will be clear from the decentralized implementation proposed in Section VI-B, the above property considerably reduces the cost and the complexity of the signaling among primary-UTs and secondary-BSs. A second benefit appears in obtaining a distributed algorithm where each primary-UT only needs to track the sum of the interferences in order to calculate the price and does not need to track the interference from each user reducing the communication and signaling costs further.

Since NNEs have favorable properties to be implemented in a decentralized fashion, we henceforth examine the computing and the uniqueness of the NNEs.

\section{ON THE UNIQUeNESS OF A NNE}

The uniqueness of a NNE for concave games with coupled constrained has been studied in [3]. In the following proposition we summarize the results useful for further developments.

Proposition 1. [3] Let

$$
\mathbf{G}(\mathbf{p})=\left(\begin{array}{cccc}
\frac{\partial^{2} u_{1}}{\partial p_{1}^{2}} & \frac{\partial^{2} u_{1}}{\partial p_{2} \partial p_{1}} & \cdots & \frac{\partial^{2} u_{1}}{\partial p_{F} \partial p_{1}} \\
\frac{\partial^{2} u_{2}}{\partial p_{1} \partial p_{2}} & \frac{\partial^{2} u_{2}}{\partial p_{2}^{2}} & \cdots & \frac{\partial^{2} u_{2}}{\partial p_{F} \partial p_{2}} \\
\vdots & \vdots & & \vdots \\
\frac{\partial^{2} u_{F}}{\partial p_{1} \partial p_{F}} & \frac{\partial^{2} u_{F}}{\partial p_{2} \partial p_{F}} & \cdots & \frac{\partial^{2} u_{F}}{\partial p_{F}^{2}}
\end{array}\right) .
$$

If the symmetric matrix $\mathbf{G}(\mathbf{p})+\mathbf{G}^{T}(\mathbf{p})$ is negative definite for all $\mathbf{p} \in \mathcal{P}$, then there exists a unique vector $\boldsymbol{\lambda}=\left(\lambda_{1}, \ldots, \lambda_{M}\right)$ and a unique NE $\mathbf{p}^{*}$ which satisfy all the KKT conditions in (7)-(9) with $\lambda_{m}^{f}=\lambda_{m}$ for all $f \in \mathcal{F}$ and $m=1, \ldots, M$, i.e., the NNE is unique ${ }^{5}$.

In order to study the uniqueness of an NNE, throughout this article, we assume that the utility set $U_{f}(\cdot)$ are twice differentiable and strictly concave. We analyze under which conditions the game $\mathcal{G}$ defined for the cognitive network with secondary-BSs as players admits a unique NNE.

Initially, we focus on the case when the interference from adjacent secondary-BSs is negligible at all the secondary-UTs, i.e. $u_{f}(\mathbf{p})=U_{f}\left(\gamma_{f}^{\prime}\right)=U_{f}\left(\frac{p_{f} h_{f}}{\sigma^{2}}\right)$. Then, $\mathbf{G}(\mathbf{p})$ is diagonal and given by

$$
\begin{array}{r}
\mathbf{G}(\mathbf{p})=\operatorname{diag}\left(\frac{h_{1}^{2}}{\sigma^{4}} U_{1}^{\prime \prime}\left(\frac{p_{1} h_{1}}{\sigma^{2}}\right)\right. \\
, \frac{h_{2}^{2}}{\sigma^{4}} U_{2}^{\prime \prime}\left(\frac{p_{2} h_{2}}{\sigma^{2}}\right), \\
\left.\ldots \frac{h_{F}^{2}}{\sigma^{4}} U_{F}^{\prime \prime}\left(\frac{p_{F} h_{F}}{\sigma^{2}}\right)\right) .
\end{array}
$$

${ }^{5}$ The condition defined in Proposition 1 is sufficient, but not necessary. In [3] a weaker sufficient condition is provided for the uniqueness of NNE. We do not consider that conditona since it is very difficult to verify in practice.
Thanks to the assumption of strict concavity of $U_{f}(\cdot)$, for $f \in \mathcal{F}$ and for every $\mathbf{p} \in \mathcal{P}$, all the diagonal elements of the matrix $\mathbf{G}(\mathbf{p})$ are strictly negative and according to Proposition $1, N N E$ is unique.

Then, we consider the case where the interference from adjacent secondary-BSs is not negligible. In this case,

$$
\begin{array}{cc}
\frac{\partial^{2} U_{f}}{\partial p_{f}^{2}}= & \kappa_{f} U_{f}^{\prime \prime}\left(\gamma_{f}\right) h_{f} \\
\frac{\partial^{2} U_{f}}{\partial p_{\ell} \partial p_{f}}= & -\kappa_{f} \widetilde{h}_{\ell}^{f}\left(\gamma_{f} U_{f}^{\prime \prime}\left(\gamma_{f}\right)+U_{f}^{\prime}\left(\gamma_{f}\right)\right)
\end{array}
$$

with $\kappa_{f}=\frac{h_{f}}{\left(\sigma^{2}+\mathbf{p}_{-f}^{T} \widetilde{\mathbf{h}}^{f}\right)^{2}}$. Then, the general expression of matrix $\mathbf{G}(\mathbf{p})$ is presented in (14) at the bottom of next page and the properties of the matrix $\mathbf{G}(\mathbf{p})$ strictly depend on the selected functions $U_{f}(\cdot)$, with $f \in \mathcal{F}$, and on the realizations of the channels $\mathbf{h}$ and $\widetilde{\mathbf{h}}^{f}, f \in \mathcal{F}$. In general, it is not clear if the matrix $\mathbf{G}(\mathbf{p})+\mathbf{G}^{T}(\mathbf{p})$ is negative definite for every $\mathbf{p} \in \mathcal{P}$ and thus, the uniqueness of the NNE is not guaranteed. Even for given functions $U_{f}(\cdot), f \in \mathcal{F}$, and channel $\mathbf{h}$ and $\widetilde{\mathbf{h}}^{f}$, it is not clear if the condition of Proposition 1 is satisfied. Nevertheless, in the next section we provide a class of utility functions which are of practical interest and possess unique NNEs when the interference at a secondary-UT from adjacent secondary-BSs is not negligible.

\section{WEAKLY NORMALIZED NE}

First of all, we state a proposition whose proof is in Appendix.

Proposition 2. Let $\mathcal{G} \equiv\left\{\mathcal{F}, \mathcal{P},\left\{U_{f}\left(\gamma_{f}\right)\right\}_{f \in \mathcal{F}}\right\}$ and $\mathcal{G}^{\prime} \equiv$ $\left\{\mathcal{F}, \mathcal{P},\left\{V_{f}\left(\gamma_{f}\right)\right\}_{f \in \mathcal{F}}\right\}$ be two games of the kind defined in (5) with identical player and strategy sets and different utility sets. Let the functions $V_{f}$ and $U_{f}, f \in \mathcal{F}$, be strictly increasing functions. Then, if $\mathbf{p}^{*}$ is a NE of game $\mathcal{G}^{\prime}$, then it is also a $N E$ for game $\mathcal{G}$.

By using the above proposition, we formally define the weakly normalized $N E$ (WNNE) of game $\mathcal{G}$ induced by the utility set $\mathcal{V}=\left\{V_{f}\left(\gamma_{f}\right)\right\}_{f \in \mathcal{F}}$.

Definition 1. Let game $\mathcal{G}^{\prime}$ with utility set $\mathcal{V} \equiv\left\{V_{f}\left(\gamma_{f}\right) \mid f \in\right.$ $\mathcal{F}\}$ and strictly increasing $V_{f}(\cdot)$ have a NNE $\overline{\mathbf{p}}$. Then, $\overline{\mathbf{p}}$ is also a $N E$ of the game $\mathcal{G}$ with utility set $\mathcal{U} \equiv\left\{U_{f}\left(\gamma_{f}\right) \mid f \in \mathcal{F}\right\}$ and strictly increasing $U_{f}(\cdot)$. This $N E \overline{\mathbf{p}}$ is be denoted as the Weakly Normalized Nash equilibrium of $\mathcal{G}$ induced by the utility set $\mathcal{V}$.

Note that the WNNE depends on the set of utility functions $\mathcal{V}$. If the game $\mathcal{G}^{\prime}$ with the specified set of utility functions $\mathcal{V}$ admits a unique NNE, then there is a unique WNNE of the game $\mathcal{G}$ induced by the utility set $\mathcal{V}$.

For some game $\mathcal{G}$ the NNE may not be unique and the computation of an NNE can be highly costly in terms of the computational complexity, while it can be possible to identify a unique WNNE induced on game $\mathcal{G}$ by a different set of utility functions with lower complexity. Thus, we can obtain a $\mathrm{NE}$ of the game that retains some of the appealing properties 
of an NNE. We enlighten the benefit in detail in Sections VI-D and VII.

In cognitive radio networks with not negligible intersecondary-network interference, the concept of WNNE can be illustrated by selecting the functions $V_{f}(x)=\log (x)$ and defining the utility set

$$
\mathcal{V}=\left\{v_{f}(\mathbf{p})=\log (\gamma)=\log \left(\frac{p_{f} h_{f}}{\sigma^{2}+\mathbf{p}_{-f}^{T} \widetilde{\mathbf{h}}^{f}}\right)\right\} .
$$

Note that it is worthwhile to consider such a utility function thanks to the following features:

- $\sum_{f \in \mathcal{F}} \log \left(\gamma_{f}\right)$ is the utility function underlying a proportionally fair SINR allocation.

- When SINR $\gg 1$, then the maximum achievable rate of each secondary-UT, shortly referred as Shannon capacity, $\log (1+\mathrm{SINR})$ can be approximated by $\log (\mathrm{SINR})$, i.e. $\log (1+\mathrm{SINR}) \approx \log (\mathrm{SINR})$.

- For certain applications (e.g. voice transmission) the utility functions increase with SINR in a logarithmic manner.

By observing that

$$
x \frac{d^{2} \log (x)}{d x^{2}}+\frac{d \log (x)}{d x}=0
$$

the matrix $\mathbf{G}(\mathbf{p})$ in (14) reduces to a diagonal matrix with strictly negative diagonal elements for every $\mathbf{p} \in \mathcal{P}$ when the utility set $\mathcal{V}$ is adopted. Thus, by Proposition 1 , the NNE $\overline{\mathbf{p}}$ is unique for the above game. We can adopt $\overline{\mathbf{p}}$ as a unique WNNE induced by the set $\mathcal{V}$ to any game $\mathcal{G}$ of the kind defined in (5) with strictly increasing utility functions $U_{f}(\cdot), f \in \mathcal{F}$. For example, we can consider the set $\mathcal{V}=\left\{V_{f}\left(\gamma_{f}(\mathbf{p})\right)=\right.$ $\left.\log \left(1+\gamma_{f}\right)\right\}$ i.e. the set of Shannon Capacity functions.

\section{Computing a Normalized Nash Equilibrium}

In this section, we present the concept of coupled constrained potential games. Subsequently, we show that there are games of the type introduced in Section III-where finding an NNE is equivalent to solving a concave potential game. We propose a distributed algorithm which converges to the unique NNE of a strictly concave potential game in Section VI-B. Subsequently, we identify a class of utility functions for which the distributed algorithm can be applied to attain the unique NNE in Sections VI-C and VI-D. Finally, in Section VI-D, we discuss the significance of obtaining an NNE as it induces a WNNE in a broad class of games.

\section{A. Constrained Concave Potential Games}

Constrained potential games have been discussed in [27] and [28]. They found application to rate and power allocation in multiple access channels (MAC). To the best of our knowledge our work is the first one to provide a relationship between potential games and NNE in a cognitive network. In contrast to [27] and [28], we consider here an interference channel which presents more challenging issues than a MAC. For example, the Shannon capacity in a MAC setting admits a potential function and this property has been widely exploited in literature. However, the same utility does not admit a potential function when there is interference at a secondary-UT from other secondary-BSs in the interference channel. Additionally, in contrast to previous works, we also identify a broad class of utility functions which admit concave potential games. Finally, we propose a distributed algorithm by leveraging on the concave potential game. This distributed algorithm enables us to implement NNE or WNNE in a distributed fashion in cognitive radio networks which were not considered in [27] and [28].

We first introduce the following definitions which we use throughout.

Definition 2. [27] A non-cooperative game $\mathcal{G}$ with utility set $\left\{u_{f}(\mathbf{p}) \mid f \in \mathcal{F}\right\}$ is an exact potential game g $^{6}$ if there exists a function $\Phi(\mathbf{p})$ such that for all $f \in \mathcal{F}$ and $\left(p_{f}, \mathbf{p}_{-f}\right)$, $\left(p_{f}^{\prime}, \mathbf{p}_{-f}\right) \in \mathcal{P}$ :

$$
\Phi\left(p_{f}, \mathbf{p}_{-f}\right)-\Phi\left(p_{f}^{\prime}, \mathbf{p}_{-f}\right)=u_{f}\left(p_{f}, \mathbf{p}_{-f}\right)-u_{f}\left(p_{f}^{\prime}, \mathbf{p}_{-f}\right) .
$$

Definition 3. [27] A potential game is called a concave potential game if the potential function $\Phi(\mathbf{p})$ is concave in $\mathbf{p} \in \mathcal{P}$. If $\Phi(\mathbf{p})$ is strictly concave, it is called strictly concave potential game.

Remark 1. [4] For a differentiable utility function $u_{f}(\cdot)$, $\Phi(\cdot)$ is a potential function of the game if and only if (iff)

$$
\frac{\partial u_{f}}{\partial p_{f}}=\frac{\partial \Phi}{\partial p_{f}} \quad \forall f \in \mathcal{F} .
$$

The utility of introducing the concave potential game is shown in the following proposition.

Proposition 3. Suppose there exists a potential function $\Phi(\mathbf{p})$ of the game $\mathcal{G}$ defined in (5) as $\mathcal{G}=\left\{\mathcal{F}, \mathcal{P},\left\{u_{f}(\mathbf{p}) \mid f \in \mathcal{F}\right\}\right\}$. The solution of the following optimization problem, referred to as CCPG, is an NNE.

$$
\begin{array}{lll}
C C P G & \text { maximize }_{\mathbf{p}} & \Phi(\mathbf{p}) \\
& \text { subject to } & \mathbf{p} \in \mathcal{P}
\end{array}
$$

If the potential function $\Phi(\mathbf{p})$ is concave, then the optimal solution of the convex optimization problem CCPG is an NNE. Note that if an NNE is unique then the solution of CCPG is the unique NNE. If a coupled constraint concave game $\mathcal{G}$ admits a potential function, in general, not every NNE can be

${ }^{6}$ Hitherto, we refer to an exact potential game as a potential game.

$$
\mathbf{G}(\mathbf{p})=\left(\begin{array}{cccc}
\kappa_{1} h_{1} & -\kappa_{1} \widetilde{h}_{2}^{1} & \ldots & -\kappa_{1} \widetilde{h}_{F}^{1} \\
-\kappa_{2} \widetilde{h}_{1}^{2} & \kappa_{2} h_{2} & \cdots & -\kappa_{2} \widetilde{h}_{F}^{2} \\
\vdots & \vdots & \ddots & \vdots \\
-\kappa_{F} \widetilde{h}_{1}^{F} & -\kappa_{F} \widetilde{h}_{2}^{F} & \ldots & -\kappa_{F} h_{F}
\end{array}\right) \odot\left(\begin{array}{cccc}
U_{1}^{\prime \prime}\left(\gamma_{1}\right) & \gamma_{1} U_{1}^{\prime \prime}\left(\gamma_{1}\right)+U_{1}^{\prime}\left(\gamma_{1}\right) & \cdots & \gamma_{1} U_{1}^{\prime \prime}\left(\gamma_{1}\right)+U_{1}^{\prime}\left(\gamma_{1}\right) \\
U_{2}^{\prime \prime}\left(\gamma_{2}\right) & \cdots & \gamma_{2} U_{2}^{\prime \prime}\left(\gamma_{2}\right)+U_{2}^{\prime}\left(\gamma_{2}\right) \\
\gamma_{2} U_{2}^{\prime \prime}\left(\gamma_{2}\right)+U_{2}^{\prime}\left(\gamma_{2}\right) & \vdots & \ddots & \vdots \\
\vdots & \gamma_{F} & \vdots \\
\gamma_{F} U_{F}^{\prime \prime}\left(\gamma_{F}\right)+U_{F}^{\prime}\left(\gamma_{F}\right) & \gamma_{F} U_{F}^{\prime \prime}\left(\gamma_{F}\right)+U_{F}^{\prime}\left(\gamma_{F}\right) & \cdots & U_{F}^{\prime \prime}\left(\gamma_{F}\right)
\end{array}\right)
$$


expressed as a solution of CCPG. However, if the potential function is concave, then, each NNE is a solution of the CCPG optimization problem since the KKT conditions are not only necessary but also sufficient for optimality for concave potential games.

\section{B. A Distributed Algorithm to Determine an NNE}

In Section VI-A, we showed that, when a coupled constrained concave game $\mathcal{G}$ has a unique NNE and admits a potential function, we can solve the potential game in order to achieve the unique NNE. When the potential function $\Phi(\cdot)$ is strictly concave and $\frac{\partial U_{f}}{\partial p_{f}}$ only depends on the local information measurable at secondary-BS $f$, i.e., $p_{f}, h_{f}, \sigma^{2} \forall f \in \mathcal{F}$, then, there exists a distributed algorithm which converges to the unique optimal solution $\mathbf{p}^{*}$ and the dual optimal solution $\lambda^{*}$. The distributed algorithm is described in the following.

\section{Algorithm DIST}

Initially primary-UT $m$ selects $\boldsymbol{\lambda}^{0} \in \mathbb{R}_{+}^{M} \backslash\{\mathbf{0}\}$ randomly ${ }^{7}$. At iteration $k+1=1,2, \ldots$, the following tasks are performed:

1) Each secondary-BS $f$ sets

$$
p_{f}^{k+1}=\underset{p_{f} \geq 0}{\operatorname{argmax}} \Phi-p_{f} \boldsymbol{\lambda}^{k T} \hat{\mathbf{h}}^{f}
$$

Then, all the secondary-BSs transmit with updated power level $\mathbf{p}^{k+1}=\left(p_{1}^{k+1}, p_{2}^{k+1}, \ldots p_{F}^{k+1}\right)$.

2) Primary-UT $m$ sets

$$
\lambda_{m}^{k+1}=\left(\lambda_{m}^{k}+\delta\left(\widehat{\mathbf{h}}_{m}^{T} \mathbf{p}_{f}^{k+1}-I_{T}\right)\right)^{+}
$$

where $\delta>0$ is a sufficiently small constant. Primary-UT $m$ reports the updated cost $\lambda_{m}^{k+1}$ to all the secondary-BSs.

Since computing an NNE is equivalent to solving the convex optimization problem CCPG by Proposition 3, thus, the convergence of Algorithm DIST follows immediately from known results in [29] and it is stated in the following:

Proposition 4. Algorithm DIST converges to the unique optimal primal solution $\mathbf{p}^{*}$ and dual solution $\boldsymbol{\lambda}^{*}$ when $\Phi(\cdot)$ is strictly concave in $\mathbf{p}$.

In algorithm DIST Secondary-BS $f$ needs to find the optimal $p_{f}$. In order to find the optimal $p_{f}$, secondary-BS $f$ needs to evaluate $\frac{\partial \Phi}{\partial p_{f}}$. Since in a potential game, $\frac{\partial \Phi}{\partial p_{f}}=\frac{\partial U_{f}}{\partial p_{f}}$, thus, a secondary-BS $f$ does not need to know the utility functions of other secondary-BSs. Equivalently we can write step 1 as

$$
p_{f}^{k+1}=\underset{p_{f} \geq 0}{\operatorname{argmax}} U_{f}\left(\gamma_{f}(\mathbf{p})\right)-p_{f} \boldsymbol{\lambda}^{k T} \hat{\mathbf{h}}^{f}
$$

In step 1 , secondary-BS $f$ needs to know $\widehat{\mathbf{h}}^{f}$. Note that $\widehat{\mathbf{h}}^{f}$ consists of the values of channel gains between secondary-BS $f$ and all primary-UTs. As discussed in Section II, SecondaryBS $f$ can obtain those values locally through sensing of pilot signal sent from the primary-UTs assuming the channel reciprocity since the primary network operates in TDD mode.

\footnotetext{
${ }^{7} \boldsymbol{\lambda}^{0}$ is initialized arbitrarily.
}

Thus, when $\frac{\partial U_{f}}{\partial p_{f}}$ only depends on $p_{f}, h_{f}, \sigma^{2}$, then secondaryBS $f$ can update its power in step 1 without any costly feedback exchange with other secondary-BSs and secondaryUTs in algorithm DIST. Thus, even though the solution of potential game CCPG requires to know all the channel gains, a secondary-BS needs not know channel gains regarding other secondary-BSs and UTs in Algorithm DIST. Hence, a secondary-BS does not need to communicate with other secondary-BSs and UTs. In the following Sections VI-C and VI-D, we show that for a wide class of utility functions concave potential games exist and $\frac{\partial U_{f}}{\partial p_{f}}$ only depends on $p_{f}, h_{f}$ and $\sigma^{2}$, i.e., for a wide class of utility functions, algorithm DIST can be applied to obtain the unique NNE.

Note that primary-UTs need to broadcast the prices $\lambda$. Thus, a cooperation from primary-UTs is required. However, we need very limited cooperation from primary-UTs. The costly channel feedback from primary-UTs to secondaryBSs is not required since secondary-BS $f$ can acquire the vector $\widehat{\mathbf{h}}^{f}$ locally as discussed in Section II. Each primaryUT only needs to track the total interference ${ }^{8}$. This tracking can be performed by using a known test signal sent by the primary-BS periodically. The primary-UT does not need to track interference from each secondary-BS. Hence, it does not need to communicate with each secondary-BS. Thus, a primary-UT is oblivious of the number of secondary-UTs, their utilities, the channel parameters and the transmitted power p. Hence, the signaling and communication cost is greatly reduced. Primary-UT $m$ is also compensated by the price $\lambda_{m}$ for per unit of interference caused by the secondary-BSs. Thus, an incentive is also provided to primary-UTs for the minimal amount of cooperation required in Algorithm DIST. Thus, the distributed algorithm DIST is readily scalable and implementable in practice.

\section{Negligible Inter-Secondary Network Interference}

In this section, we show that when the interference from other secondary-BSs at each secondary-UT is negligible , then the game is a strictly concave potential game.

In this setting, SINR reduces to the SNR $\gamma_{f}^{\prime}(\mathbf{p})=$ $\frac{p_{f} h_{f}}{\sigma^{2}}, \forall f \in \mathcal{F}$. Next lemma shows that in this scenario every game $\mathcal{G}$ defined in (5) is a strictly concave potential game.

Lemma 1. Let $\Phi(\mathbf{p})=\sum_{f} U_{f}\left(\gamma_{f}^{\prime}(\mathbf{p})\right)$. Then, $\Phi(\mathbf{p})$ is a potential function for game $\mathcal{G}$ defined in (5) with utility set $\left\{U_{f}\left(\gamma_{f}^{\prime}\right) \mid f \in \mathcal{F}\right\}$. Moreover, if $U_{f}(\cdot)$ is strictly concave, then so $\Phi(\cdot)$ is.

If we focus on strictly concave functions $U_{f}(\cdot)$, the NNE is unique as we have shown in Section IV. We can evaluate the unique NNE by solving the potential game CCPG by Proposition 3. Moreover, we can apply Algorithm DIST, which will converge to optimal $\mathbf{p}^{*}$ and dual variable $\lambda^{*}$ since $\gamma_{f}^{\prime}$ is only a function of $p_{f}, h_{f}$ and $\sigma^{2}$ and $U_{f}(\cdot)$ is a function of $\gamma_{f}^{\prime}$

\footnotetext{
${ }^{8}$ It is reasonable to assume that the interference from secondary-BSs situated at far-off locations from a primary-BS is negligible in order to avoid communication overhead.
} 
in this setting. It is worth noting that the NNE in this setting also maximizes the sum of the utility functions of players under the set of constraints $\mathcal{P}$ since $\Phi=\sum_{f=1}^{F} U_{f}$.

\section{Presence of Inter-Secondary Network Interference}

When the interference from adjacent secondary-BSs is not negligible at all the secondary-UTs, then we have already seen that an NNE may be not unique. However, in Section V we have identified a utility set $\mathcal{V}$ defining a game $\mathcal{G}$, whose NNE is unique and can be used to define a unique WNNE for games with different utility sets. In this section, we show that the game with utility set $\mathcal{V}$ defined in (15) admits a potential function.

Let us consider again the utility set $\mathcal{V}$ defined in (15). Then, $V_{f}(\mathbf{p})$ is a strictly increasing concave function in $p_{f}$. The following lemma shows that the game $\mathcal{G}$ with the utility set $\mathcal{V}$ defined in (15) is a potential game.

Lemma 2. Let $\Phi(\mathbf{p})=\sum_{f} \log \left(p_{f}\right) . \Phi(\mathbf{p})$ is a potential function for the game $\mathcal{G}$ defined in (5) and the utility set (15). Moreover, the potential game is strictly concave.

Hence, the solution of CCPG will provide the unique NNE to game $\mathcal{G}$ defined in (5) with the utility set (15). Note that though $U_{f}$ depends on $\mathbf{p}_{-f}$ and $\widetilde{\mathbf{h}}^{f}, \frac{\partial \Phi}{\partial p_{f}}$ or $\frac{\partial U_{f}}{\partial p_{f}}$ only depends on $p_{f}, h_{f}$ and $\sigma^{2}$, thus, we can use Algorithm DIST described in Section VI-B to obtain the unique NNE for a game $\mathcal{G}$ with utility set (15). Note that the NNE may not maximize the sum of the utility functions of players unlike the NNEs of the games discussed in Section VI-C.

Now, we show that, in general, a potential game does not exist in this setting by using the following result.

Proposition 5. [4] For twice continuously differentiable utility functions $u_{f}, u_{\ell}, f, \ell \in \mathcal{F}$, there exists a potential function iff $\frac{\partial^{2} u_{f}}{\partial p_{\ell} \partial p_{f}}(\mathbf{p})=\frac{\partial^{2} u_{\ell}}{\partial p_{f} \partial p_{\ell}}(\mathbf{p}), \forall \mathbf{p} \in \mathcal{P}$.

It is easy to verify from (13) that in general the utilities $U_{f}\left(\gamma_{f}\right)$ do not satisfy the conditions stated in Proposition 5. Thus, in general a potential function does not exist. There exist large classes of function $U_{f}\left(\gamma_{f}(\mathbf{p})\right)$ which are strictly concave functions of $p_{f}$ but still they do not admit a potential function. One such examples is $U_{f}\left(\gamma_{f}\right)=\log \left(1+\gamma_{f}\right)$.

For all these cases when the functions $U_{f}(\cdot)$ are strictly increasing, it is convenient to invoke to Proposition 2 and resort to the concept of WNNE with respect to the utility set $\mathcal{V}$ in (15). We have already shown in Lemma 2 that the game $\mathcal{G}$ with utility set $\mathcal{V}$ in (15) is a strictly concave potential game and we can attain the unique NNE using Algorithm DIST proposed in Section VI-B. Hence, we can easily obtain the WNNE for any game $\mathcal{G}$ including the one where $U_{f}\left(\gamma_{f}\right)=\log \left(1+\gamma_{f}\right)$ even though this latter game is not a potential game.

\section{A Relevant CAse}

In this section we focus on the Shannon capacity as utility, i.e. $U_{f}\left(\gamma_{f}\right)=B \log \left(1+\gamma_{f}\right), B$ being the channel frequency bandwidth. First, we determine conditions which are sufficient to conclude about uniqueness of a NNE. In Section VI-D we saw that there is no potential function for this game. Thus, we cannot utilize the results regarding potential games in order to obtain the NNE. Nevertheless, we provide an algorithm which returns the unique NNE for a system with a single primaryUT when the uniqueness condition is satisfied and under some technical conditions detailed throughout this section.

Let us start introducing a first assumption that guarantees the uniqueness of the NNE.

Assumption 1. The following matrix is row wise and column wise diagonally dominant

$$
\bar{H}=\left[\begin{array}{cccc}
k_{1} h_{1} & k_{1} \tilde{h}_{2}^{1} & \ldots & k_{1} \tilde{h}_{F}^{1} \\
\ldots & \ldots & \ldots & \ldots \\
k_{F} \tilde{h}_{1}^{F} & k_{F} \tilde{h}_{2}^{F} & \ldots & k_{F} h_{F}
\end{array}\right]
$$

for every $k_{f}, f \in \mathcal{F}$ such that $\frac{B h_{f}}{\left(\bar{p}_{f} h_{f}+\overline{\mathbf{p}}_{-f}^{T} \widetilde{\mathbf{h}}^{f}+\sigma^{2}\right)^{2}} \leq k_{f} \leq$ $\frac{B h_{f}}{\sigma^{4}}$ where $\bar{p}_{f}=\min _{m} \frac{I_{T}}{\hat{h}_{m}^{f}}, f \in \mathcal{F}$.

Remark 2. Note that the following conditions are sufficient to conclude that the matrix $\bar{H}$ is row wise and column wise diagonally dominant:

$$
\begin{aligned}
& h_{f}>\mathbf{1}_{F-1}^{T} \widetilde{\mathbf{h}}^{f} \\
& \frac{B h_{f}^{2}}{\left(\bar{p}_{f} h_{f}+\overline{\mathbf{p}}_{-f}^{T} \widetilde{\mathbf{h}}^{f}+\sigma^{2}\right)^{2}}>\sum_{\ell \neq f} \frac{B h_{\ell} \widetilde{h}_{f}^{\ell}}{\sigma^{4}} .
\end{aligned}
$$

Though the above conditions are technical but the above conditions are satisfied for sufficiently small $\widetilde{h}_{f}^{\ell}$ and $\widetilde{h}_{\ell}^{f}, \ell \neq f$ compared to $h_{f}$.

The following lemma shows that if Assumption 1 holds, then the NNE is unique.

Lemma 3. If Assumption 1 holds, then there exists a unique $N N E$.

Henceforth, we assume that Assumption 1 is satisfied and we examine how to compute the unique NNE. Since there is a unique NNE $\mathbf{p}^{*}=\left(p_{1}, \ldots, p_{F}\right) \in \mathcal{P}$, thus there is a unique $\boldsymbol{\lambda} \geq 0$ satisfying the system of equations (9) and the complementary slackness equations

$$
\lambda_{m}\left(\mathbf{p}^{* T} \widehat{\mathbf{h}}_{m}-I_{T}\right)=0 \quad \forall m=1, \ldots, M .
$$

By simple algebraic calculation we obtain the optimal $p_{f}^{*}$ for a given $\lambda$ :

$$
p_{f}^{*}=\frac{1}{h_{f}}\left(\frac{B h_{f}}{\boldsymbol{\lambda}^{T} \widehat{\mathbf{h}}^{f}}-\sigma^{2}-\mathbf{p}_{-f}^{T} \widetilde{\mathbf{h}^{f}}\right)^{+} .
$$

The optimal strategy of a secondary-BS depends on the strategies adopted by the other secondary-BSs and $\boldsymbol{\lambda}$. It is not known a priori which $p_{f}^{*}$ is zero and which constraints are active, i.e. which components of $\boldsymbol{\lambda}$ are strictly positive. The solution requires us to consider all the possible combinations. Thus, it is computationally demanding to compute the unique NNE. The complexity of the problem reduces to some extent when we focus on the scenario with one single primary-UT, 
i.e., $M=1$. Then, we consider such a scenario. Since there is only one constraint with a slight abuse of notation we shortly denote $\hat{h}_{1}^{f}$ as $\hat{h}^{f} \forall f \in \mathcal{F}$ and $\widehat{\mathbf{h}}_{1}$ as $\widehat{\mathbf{h}}$.

The following observation shows that the single constraint must be active at the NNE in this setting.

Observation 1. At NNE $\mathbf{p}$, we must have $\mathbf{p}^{T} \widehat{\mathbf{h}}=I_{T}$.

Since there is a single constraint, thus (22) reduces to

$$
p_{f}^{*}=\frac{1}{h_{f}}\left(\frac{B h_{f}}{\lambda \hat{h}^{f}}-\mathbf{p}_{-f}^{* T} \widetilde{\mathbf{h}}^{f}-\sigma^{2}\right)^{+}
$$

From Observation 1 we also must have

$$
\mathbf{p}^{T} \widehat{\mathbf{h}}=I_{T} .
$$

Then, the NNE $\mathbf{p}^{*}$ and the corresponding $\lambda$ have to satisfy (23) and (24).

In the following, we provide an algorithm yielding $\mathbf{p}^{*}$ for a given $\lambda$. Let

$$
\mathbf{H}=\left[\begin{array}{cccc}
h_{1} & \tilde{h}_{2}^{1} & \ldots & \tilde{h}_{F}^{1} \\
\cdot & \cdot & \ldots & \cdot \\
\tilde{h}_{1}^{F} & \tilde{h}_{2}^{F} & \ldots & h_{F}
\end{array}\right]
$$

and

$$
\overline{\mathbf{h}}=\left(\begin{array}{ccc}
\frac{B h_{1}}{\hat{h}^{1}} & \cdots & \frac{B h_{F}}{\hat{h}^{F}}
\end{array}\right)^{T}
$$

We assume that

Assumption 2. The matrix $\mathbf{H}$ is row wise and column wise strictly diagonally dominant.

Remark 3. Since $\mathbf{H}$ is row wise and column wise strictly diagonally dominant, thus the square matrix $\mathbf{H}_{K}$ is invertible for any index subset $K$ of $\mathcal{F}$.

Under Assumptions 1 and 2, we can state the following algorithm.

\section{Algorithm OPTI:}

Given $\lambda>0$, we execute the following steps:

1) Initialize $K^{0}$ to the empty set, i.e. set $K^{0}=\emptyset$. For each index $i \in \mathcal{F}$, if $\frac{B h_{i}}{\lambda \hat{h}^{i}} \leq \sigma^{2}$ set $p_{i}^{*}=0$ otherwise assign $K^{0} \leftarrow K^{0} \bigcup\{i\}$.

2) If $K^{0}$ is empty exit; otherwise go to the next step.

3) Let $I=0$.

4) Solve the system of equations ${ }^{9}$

$$
\mathbf{H}_{K^{I}} \mathbf{p}_{K^{I}}=\frac{1}{\lambda} \overline{\mathbf{h}}_{K^{I}}-\sigma^{2} \mathbf{1}_{\left|K^{I}\right|}
$$

and assign the solution to $\mathbf{p}_{K^{I}}^{0}$.

5) If $\mathbf{p}_{K^{I}}^{0} \succeq \mathbf{0}$, then set $\mathbf{p}_{K^{I}}^{*}=\mathbf{p}_{K^{I}}^{0}$ and exit. Otherwise go to the next step.

6) Assign $I \leftarrow I+1$ and set $K^{I}=\emptyset$. For each index $i \in K^{I-1}$, if $p_{i}^{0}<0$ set $p_{i}^{*}=0$ otherwise assign $K^{I} \leftarrow$ $K^{I} \bigcup\{i\}$ and go to step 4 .

First note that if $K^{0}$ is empty, then $\mathbf{p}_{i}^{*}=\mathbf{0}_{F}$ is the only possible solution. Additionally, the algorithm stops at most after $F$ iterations. Thus, the algorithm scales linearly with the

\footnotetext{
${ }^{9}$ Since $\mathbf{H}_{K^{0}}$ is invertible, the solution is linear and the solution unique.
}

number of secondary-BSs. Finally, if $i \notin K^{I}$ at some iteration $I$, then $p_{i}^{*}=0$.

Algorithm OPTI converges to the desired $\mathbf{p}^{*}$ under the following assumptions and this property is stated in Proposition 6.

Assumption 3. Fix any index $k$. For any $i=1, \ldots, k-1, k+$ $1, \ldots, F$, we have

$$
\sum_{j \neq k, i} \frac{\tilde{h}_{j}^{k} \tilde{h}_{i}^{j}}{h_{j}} \leq \tilde{h}_{i}^{k}
$$

and

$$
\sum_{j \neq k} \frac{\tilde{h}_{j}^{k} \tilde{h}_{k}^{j}}{h_{k} h_{j}} \leq 1
$$

Proposition 6. For a given $\lambda>0$, Algorithm OPTI converges to $\mathrm{p}^{*}$, the solution to the system of equations (23) for every $f \in \mathcal{F}$.

Now, we present Algorithm DIST-INT to update $\lambda$ in a suitable way to attain the NNE $\overline{\mathbf{p}}$ and the corresponding $\lambda$. In the following, we denote by $\mathbf{p}^{*}(\lambda)$ the result of Algorithm OPTI for a given $\lambda$.

\section{Algorithm DIST-INT:}

1) Set the accuracy $\epsilon$ and the step size $\delta$ to desired positive values. Initialize $\lambda^{0}$ to positive value ${ }^{10}$ and set $J=0$.

2) Apply Algorithm OPTI to determine $\mathbf{p}^{*}\left(\lambda^{J}\right)$.

3) Assign $J \rightarrow J+1$ and set

$$
\lambda^{J}=\left(\lambda^{(J-1)}+\delta\left(\widehat{\mathbf{h}}^{T} \mathbf{p}^{*}\left(\lambda^{J-1}\right)-I_{T}\right)\right)^{+}
$$

where $\delta>0$ is a constant step size.

4) If $\left|\widehat{\mathbf{h}}^{T} \mathbf{p}^{*}\left(\lambda^{J-1}\right)-I_{T}\right| \leq \epsilon$, then exit, otherwise go to step 2.

The primary-UT only needs to track the total interference like in Algorithm DIST. The primary-UT does not need to track individual interference from a secondary-BS. Also note that primary-UT is compensated with $\lambda$ to provide an incentive to cooperate during the convergence of Algorithm DIST-INT. Hence, computation and signaling costs between primary-UTs and secondary-BSs are greatly reduced.

Note that $p_{i}^{*}$ is a continuous decreasing function of $\lambda$ if the interference from other players is small. From (23) it is clear that for a sufficiently small $\lambda$, at least one $p_{i}^{*}$ will be sufficiently large such that $p_{i}^{*} \hat{h}^{i}>I_{T}$. On the other hand, for sufficiently large $\lambda$, for all $i \in \mathcal{F} p_{i}^{*}=0$ and thus $\widehat{\mathbf{h}}^{T} \mathbf{p}^{*}(\lambda)<I_{T}$. Thus, by the intermediate theorem of continuity, there exists surely a $\lambda>0$ such that $\widehat{\mathbf{h}}^{T} \mathbf{p}^{*}(\lambda)=I_{T}$. Hence, we obtain the following result.

Proposition 7. If Assumption 1 holds, then for small enough $\delta$, Algorithm DIST-INT converges to an optimal $\lambda$, which in turn yields an NNE $\overline{\mathbf{p}}$ by Algorithm OPTI.

Discussion: Algorithm DIST-INT converges to the unique NNE with a single primary-UT and when Assumptions 13 are satisfied. Hence, if there are multiple primary-UTs or

\footnotetext{
${ }^{10}$ In the case it is necessary to avoid that the constraints on the communications are violated in during the transient of the algorithm, then $\lambda^{0}$ has to be initialized to a sufficiently high value.
} 


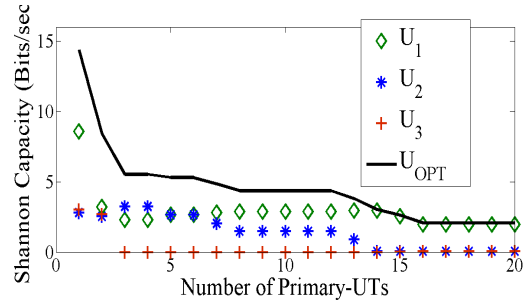

Fig. 3. $U_{O P T}$ and $U_{f}, f=1,2,3$ versus number of primary-UTs for Shannon capacity and $F=3$.

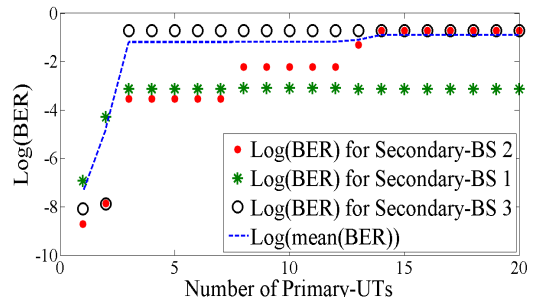

Fig. 4. $\log (\mathrm{BER})$ versus number of primary-UTs for $F=3$.

Assumptions 1-3 are not satisfied, we have to leverage on the concept of WNNE as explained in Section V.

Additionally, in Algorithm OPTI either each secondary-BS needs to know the channel parameters $\mathbf{H}$ and $\tilde{\mathbf{h}}^{f}$ or we require a central controller which has the above information and coordinates among secondary-BSs. Thus, the signaling and communication cost increases in Algorithm OPTI compared to Algorithm DIST. However, WNNE can be implemented using Algorithm DIST where secondary-BSs do not need to exchange information among themselves. Hence, we can leverage on WNNE if we want to reduce the signaling and communication cost.

\section{NumERICAL RESUlTS}

We numerically evaluate the characteristics of an NNE strategy profile for several utility functions. We consider two scenarios: i) The interference at each secondary-UT from adjacent secondary-BSs is negligible $\left(\gamma_{i} \approx \mathrm{SNR}_{i}\right)$, ii) The interference is not negligible $\left(\gamma_{i}=\operatorname{SINR}_{i}\right)$.

To generate $\hat{\mathbf{h}}_{m}^{i}, \tilde{\mathbf{h}}_{f}^{i}$, we first randomly place secondary-BSs and primary-UTs in a disc of radius $r_{1}$. Then, we randomly place a secondary-UT in a disc of radius $r_{2}$ around each secondary-BS as in Fig. 1. We take $r_{1}>r_{2}$ because in practice, a secondary-UT is in a close vicinity of its secondaryBS compared to the range of primary-BS as a secondary-BS
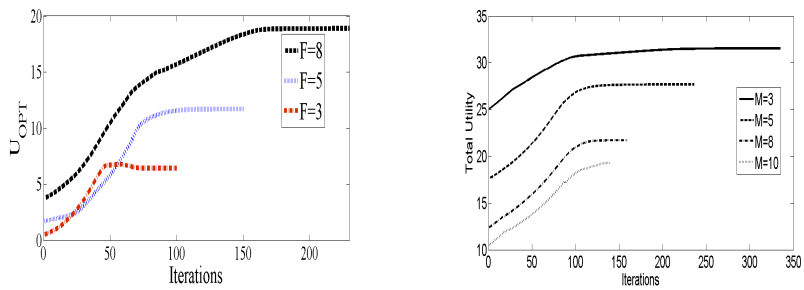

Fig. 5. Convergence analysis of Al- Fig. 6. Convergence analysis of Algorithm DIST for $F=3,5$ and 8 gorithm DIST versus the number of secondary-BSs with $M=5$. primary-UTs for $F=3$. has smaller coverage area compared to a primary-BS. Since we consider that primary-UTs and secondary-BSs are placed randomly, our numerical analysis also includes the possible setting where secondary-BSs and primary-UTs may be close to each other. We compute the channel gain between two nodes according to the formula: $K d^{-\beta}$ where $K$ is a parameter which depends on the frequency, the antenna gains of the transmitter and the receiver $d$ is the distance between two entities and $\beta$ is the path loss exponent. Similar simulation setup has been considered in several papers including [2], [14], [21]. For all simulations we take $r_{1}=20, K=1, \beta=2$ and $\sigma^{2}=1$. We consider $r_{2}=2$ for all simulations except for simulation in Fig. 9. Throughout this section, we use the following notations:

$$
\begin{aligned}
U_{\mathrm{OPT}} & =\max _{\mathbf{p} \in \mathcal{P}} \sum_{f \in \mathcal{F}} U_{f}\left(\gamma_{f}(\mathbf{p})\right) \\
U_{\mathrm{NNE}} & =\sum_{f \in \mathcal{F}} U_{f}\left(\gamma_{f}\left(\mathbf{p}_{N N E}\right)\right)
\end{aligned}
$$

where $\mathbf{p}_{N N E}$ is the NNE strategy profile.

\section{A. Negligible Interference at secondary-UT from adjacent secondary-BSs}

In this setting $\gamma_{i}=\gamma_{i}^{\prime}=\frac{p_{i} h_{i}}{\sigma^{2}}$.

We consider the following utility functions

1) Shannon Capacity: Here $U_{f}\left(\gamma_{f}^{\prime}\right)=B \log \left(1+\gamma_{f}^{\prime}\right)$.

2) Bit Error Rate: From [23] we can approximate bit error rate (BER) for $K$-QAM modulation at secondary-BS $f$ as follows:

$$
\mathrm{BER}=0.2 \exp \left(-\frac{3 \gamma_{f}^{\prime}}{2(K-1)}\right) .
$$

Since each secondary-BS wants to minimize the BER, we define the utility function as

$$
U_{f}\left(\gamma_{f}^{\prime}\right)=-0.2 \exp \left(-\frac{3 \gamma_{f}^{\prime}}{2(K-1)}\right) .
$$

The above utility function is strictly concave in $\gamma_{f}^{\prime}$ and thus, the NNE is unique.

We set $I_{T}=5 d B, B=1 \mathrm{MHz}$ and assume a 4-QAM modulation for all simulations in this subsection. Recall from Section VI-C that $\mathbf{p}_{N N E}$ is also the optimal solution for $U_{O P T}$ i.e. $U_{O P T}=U_{N N E}$.

First, we study the variation of maximum achievable total utility $U_{O P T}$ when the Shanon capacity is the utility function with the number of primary-UTs. Fig. 3 shows that, as the number of primary-UTs increases, $U_{O P T}$ and the individual utilities decrease. Intuitively, when the number of primary-UTs increases the strategy set $\mathcal{P}$ reduces. The decrement of $U_{O P T}$ becomes small as the number of primary-UTs increases. Thus, an increase in the number of primary-UTs does not affect the individual utility significantly when it exceeds a certain threshold.

In Fig. 4 we adopt as utility function the BER in (28). Fig. 4 reveals that the mean BER and each secondary-BS's BER increase as the number of primary-UTs increases since each 


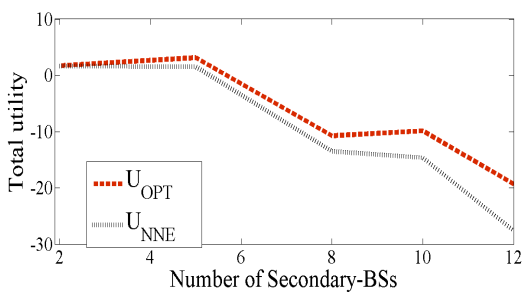

Fig. 7. $U_{O P T}$ and $U_{N N E}$ versus number of secondary-BSs with $M=5$.

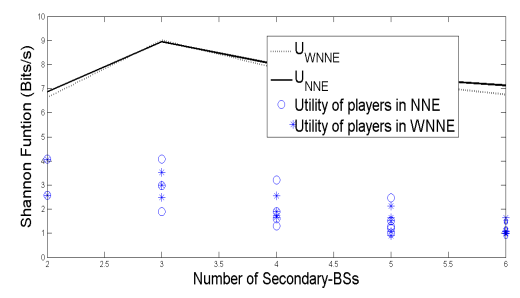

Fig. 8. $U_{W N N E}, U_{N N E}$ and individual utilities versus the number of secondary-BSs for $I_{T}=$ $10 \mathrm{~dB}$.

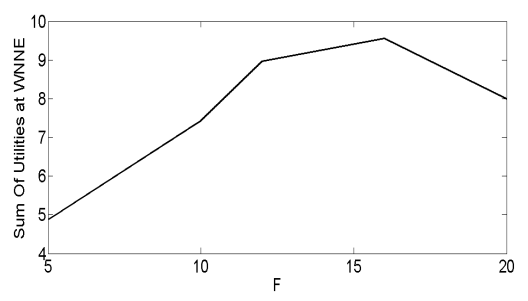

Fig. 9. The sum of the utilities of SecondaryBSs at WNNE with $M=10 \& I_{T}=10 \mathrm{~dB}$.
secondary-BS transmits with lower power as the number of primary-UTs increases. The rate of its increment slows down as the number of primary-UTs increases. Intuitively, as the number of primary-UTs increases, the strategy set $\mathcal{P}$ remains almost identical. Thus, the power remains almost the same even when the number of primary-UTs increases.

Fig. 5 shows the convergence of Algorithm DIST for systems with different number of secondary-BSs, $F=3,5$, and 8 . Numerical computations reveal that the convergence rate is higher for smaller number of secondary-BSs. Fig. 6 shows the convergence of Algorithm DIST for systems with different number of primary-UTs. Numerical analysis reveals that the convergence rate increases as the number of primary-UTs increase since it decreases the strategy space $\mathcal{P}$.

\section{B. Non-Negligible Interference at Secondary-UTs from Adja- cent Secondary-BSs}

When we adopt the following utility $U_{f}\left(\gamma_{f}\right)=\log \left(\gamma_{f}\right), f \in$ $\mathcal{F}$, then it is easy to verify that the maximization of $\sum_{i} U_{i}\left(\gamma_{i}\right)$ for $\mathbf{p} \in \mathcal{P}$ is a geometric programming [30]. Hence, we can employ standard optimization tools to compute $U_{\mathrm{OPT}}$.

Fig. 7 reveals that as the number of secondary-BSs increases the difference between $U_{\mathrm{OPT}}$ and $U_{\mathrm{NNE}}$ increases. Intuitively, when the number of secondary-BSs is small, then the interference at a secondary-UT is not significant and $U_{\mathrm{NNE}}$ closely matches $U_{\mathrm{OPT}}$. However, as the number of secondary-BSs increases, $U_{\mathrm{NNE}}$ decreases and the difference between $U_{\mathrm{NNE}}$ and $U_{\mathrm{OPT}}$ increases. Note that $U_{\mathrm{OPT}}$ also decreases with the number of secondary-BSs. Intuitively, as $U_{f}\left(\gamma_{f}\right)=\log \left(\gamma_{f}\right)$, it must be $p_{f}>0$ for any $f \in \mathcal{F}^{11}$. Thus, as the number of secondary-BSs increases the interference at a secondary-UT becomes significant as each additional secondary-BS transmits with nonzero power. Thus, $U_{O P T}$ decreases with the number of secondary-BSs as the interference at a secondary-UT from other secondary-BSs becomes significant.

Shannon capacity: We also numerically evaluate the characteristics of the NNE strategy profile when secondary-BS $f$ 's utility is $U_{f}\left(\gamma_{f}\right)=B \log \left(1+\gamma_{f}\right)$.

We obtain the NNE using the algorithms OPTI and DISTINT. We consider $B=1, \delta=0.01, \lambda^{0}=5$ and $\epsilon=5 \times$ $10^{-4}$. Assumptions 1-3 are satisfied for most of the randomly generated instances of the system for $F \leq 8$. Let $U_{\mathrm{WNNE}}$ denote the total utility at a WNNE $\mathbf{p}_{W N N E}$, i.e., $\mathbf{p}_{W N N E}$ is the NNE for a game with utility function $U_{f}\left(\gamma_{f}\right)=\log \left(\gamma_{f}\right)$. Fig.8 reveals that $U_{\mathrm{WNNE}}$ closely matches $U_{\mathrm{NNE}}$ when the

${ }^{11}$ Otherwise, $U_{O P T}$ would be negative infinity. number of secondary-BSs is very small. But as the number of secondary-BSs increases the difference between $U_{\mathrm{NNE}}$ and $U_{\mathrm{WNNE}}$ increases since the interference at a secondary-UT from other secondary-BSs increases. Fig. 8 also shows that the fluctuation of the utilities across players is higher for power allocations based on the NNE. Intuitively, $\sum_{f} \log \left(\gamma_{f}\right)$ induces a proportional fair SINR allocation, thus the utilities at the WNNE vary on a relatively smaller range compared to the utilities corresponding to the power allocation based on an NNE.

Large $r_{2}$ and large $F$ : Finally, Fig. 9 shows the $U_{\mathrm{WNNE}}$ as the number of secondary-BSs increase. We still consider the Shannon capacity function as the utility function. We consider $r_{2}=8$, hence, it models the setting where the coverage area of secondary-BS is large. We consider the number of primaryUTs $(M)$ as 10 . Note that, the NNE may not be unique in this setting since Assumption 1 may not be satisfied. Additionally, we cannot use Algorithm DIST-INT since $M>1$. Instead, we can obtain WNNEs using Algorithm DIST. Fig. 9 reveals that the sum of the utilities $\left(U_{\mathrm{WNNE}}\right)$ increases initially with the number of secondary-BSs, but it decreases after it reaches a certain threshold. Intuitively, the characteristic of $U_{\mathrm{WNNE}}$ is similar to the characteristic observed in Fig. 7 since $\boldsymbol{p}_{\mathrm{WNNE}}$ is a NNE when the utility function is $\log \left(\gamma_{f}\right)$.

\section{CONCLUSION}

We investigated the power allocation problem in cognitive radio networks using a game theoretic setting. Each secondaryBS selects a transmit power subject to the global constraint that the total interference should be below a given threshold at each primary-UT. This game falls into the category of the coupled constrained concave games. We adopted the NNE as an equilibrium concept since it caters to distributed settings. We showed that the NNE is unique when the interference at a secondary-UT from adjacent secondary-BSs is negligible. But an NNE may not be unique when the interference at a secondary-UT from adjacent secondary-BSs is not negligible. We identified a class of utility functions for which the NNE is unique in the latter setting. We also proposed a distributed algorithm which converges to the unique NNE for those utility functions. In the distributed algorithm, secondary-BSs do not exchange information among themselves and each primaryUT only needs to track the total interference. When it is computationally difficult to obtain an NNE or its uniqueness cannot be guaranteed, we leveraged on the concept of WNNE as an equilibrium concept which retains most of the properties of the NNE but it can be obtained with lower complexity 
compared to NNE. We showed the importance of WNNE when interference at a secondary-UTs from adjacent secondary-BSs is not negligible and the utility function is Shannon Capacity function.

We assumed that a secondary-BS knows the exact values of channel parameters for the channel between the primaryUTs. The characterization of NNE when secondary-BSs do not know the exact values of those channel parameters is a work for the future.

\section{REFERENCES}

[1] A. Ghosh, L. Cottatellucci, and E. Altman, "Normalized Nash equilibrium for power allocation in femto base stations in heterogeneous network," in 13th International Symposium on Modeling and Optimization in Mobile, Ad Hoc, and Wireless Networks (WiOpt), Mumbai , India, May 2015. [Online]. Available: https://hal.inria.fr/ hal-01134133

[2] L. B. Le and E. Hossain, "Resource allocation for spectrum underlay in cognitive radio networks," Wireless Communications, IEEE Transactions on, vol. 7, no. 12, pp. 5306-5315, December 2008.

[3] J. Rosen, "Existence and uniqueness of equilibrium points for concave N-person games," Econometrica, vol. 33, no. 3, pp. 520-534, July 1965.

[4] D. Monderer and L. Shapley, "Potential games," Games and Economic Behavior, vol. 14, pp. 124-143, 1996.

[5] W. S. T. B. Zhu Han, Dusit Niyato and A. Hjorungnes, Game Theory in Wireless and Communication Networks: Theory, Models, and Applications. Cambridge University Press, New York, 2012.

[6] A. Attar, M. Nakhai, and A. Aghvami, "Cognitive radio game for secondary spectrum access problem," Wireless Communications, IEEE Transactions on, vol. 8, no. 4, pp. 2121-2131, April 2009.

[7] A. T. Hoang, Y.-C. Liang, and M. Islam, "Power control and channel allocation in cognitive radio networks with primary users' cooperation," Mobile Computing, IEEE Transactions on, vol. 9, no. 3, pp. 348-360, March 2010

[8] H. Yu, L. Gao, Z. Li, X. Wang, and E. Hossain, "Pricing for uplink power control in cognitive radio networks," Vehicular Technology, IEEE Transactions on, vol. 59, no. 4, pp. 1769-1778, May 2010.

[9] D. I. Kim, L. B. Le, and E. Hossain, "Joint rate and power allocation for cognitive radios in dynamic spectrum access environment," Wireless Communications, IEEE Transactions on, vol. 7, no. 12, pp. 5517-5527, December 2008

[10] A. Al Daoud, T. Alpcan, S. Agarwal, and M. Alanyali, "A stackelberg game for pricing uplink power in wide-band cognitive radio networks," in Decision and Control, 2008. CDC 2008. 47th IEEE Conference on, Dec 2008, pp. 1422-1427.

[11] M. Razaviyayn, Z.-Q. Luo, P. Tseng, and J.-S. Pang, "A Stackelberg game approach to distributed spectrum management," Mathematical Programming, vol. 129, no. 2, pp. 197-224, 2011.

[12] S. Guruacharya, D. Niyato, D. I. Kim, and E. Hossain, "Hierarchical competition for downlink power allocation in OFDMA femtocell networks," Wireless Communications, IEEE Transactions on, vol. 12, no. 4 pp. 1543-1553, 2013.

[13] Y. Su and M. van der Schaar, "A new perspective on multi-user power control games in interference channels," Wireless Communications, IEEE Transactions on, vol. 8, no. 6, pp. 2910-2919, June 2009.

[14] C. Yang, J. Li, and Z. Tian, "Optimal power control for cognitive radio networks under coupled interference constraints: A cooperative game-theoretic perspective," Vehicular Technology, IEEE Transactions on, vol. 59, no. 4, pp. 1696-1706, May 2010.

[15] J. Huang, R. Berry, and M. Honig, "Distributed interference compensation for wireless networks," Selected Areas in Communications, IEEE Journal on, vol. 24, no. 5, pp. 1074-1084, May 2006.

[16] P. Semasinghe, E. Hossain, and K. Zhu, "An evolutionary game for distributed resource allocation in self-organizing small cells," Mobile Computing, IEEE Transactions on, vol. 14, no. 2, pp. 274-287, Feb 2015.

[17] M. Bennis, S. Guruacharya, and D. Niyato, "Distributed learning strategies for interference mitigation in femtocell networks," in Global Telecommunications Conference (GLOBECOM 2011), 2011 IEEE, Dec 2011, pp. 1-5.
[18] Y. Xing, C. Mathur, M. Haleem, R. Chandramouli, and K. Subbalakshmi, "Dynamic spectrum access with QoS and interference temperature constraints," Mobile Computing, IEEE Transactions on, vol. 6, no. 4, pp. 423-433, April 2007.

[19] Y. Wu and D. Tsang, "Distributed power allocation algorithm for spectrum sharing cognitive radio networks with QoS guarantee," in INFOCOM 2009, IEEE, April 2009, pp. 981-989.

[20] H. Li, Y. Gai, Z. He, K. Niu, and W. Wu, "Optimal power control game algorithm for cognitive radio networks with multiple interference temperature limits," in Vehicular Technology Conference, 2008. VTC Spring 2008. IEEE, May 2008, pp. 1554-1558.

[21] F. Wang, M. Krunz, and S. Cui, "Price-based spectrum management in cognitive radio networks," Selected Topics in Signal Processing, IEEE Journal of, vol. 2, no. 1, pp. 74-87, Feb 2008.

[22] A. A. Kulkarni and U. V. Shanbhag, "On the variational equilibrium as a refinement of the generalized nash equilibrium," Automatica, vol. 48, no. 1 , pp. $45-55,2012$.

[23] A. Galindo-Serrano, E. Altman, and L. Giupponi, "Equilibrium selection in interference management non-cooperative games in femtocell networks," in Performance Evaluation Methodologies and Tools (VALUETOOLS), 2012 6th International Conference on. IEEE, Oct 2012, pp. 198-203.

[24] I. Akyildiz, W. Lee, M. Vuran, and S. Mohanty, " Next Generation/Dynamic spectrum access/cognitive radio wireless networks: A survey," Computer Networks, vol. 50, no. 3, pp. 2127-2159, 2006

[25] Q. Zhao, S. Geirhofer, L. Tong, and B. Sadler, "Opportunistic spectrum access via periodic channel sensing," Signal Processing, IEEE Transactions on, vol. 56, no. 2, pp. 785-796, Feb 2008.

[26] S. Boyd and L. Vandenberghe, Convex optimization. Cambridge University Press, New York, 2007.

[27] Q. Zhu, "A Lagrangian approach to constrained potential games: Theory and examples," in Decision and Control, 2008. CDC 2008. 47th IEEE Conference on, Dec 2008, pp. 2420-2425.

[28] G. Scutari, S. Barbarossa, and D. Palomar, "Potential games: A framework for vector power control problems with coupled constraints," in Acoustics, Speech and Signal Processing, 2006. ICASSP 2006 Proceedings. 2006 IEEE International Conference on, vol. 4, May 2006, pp. IV-241-IV-244.

[29] D. Bertsekas and J. Tsitsiklis, Parallel and distributed computation: Numerical methods. Athena Scientific, 1975.

[30] S. Boyd, S.-J. Kim, L. Vandenberghe, and A. Hassibi, "A tutorial on geometric programming," Optimization and engineering, vol. 8, no. 2, pp. $67-127,2007$.

\section{APPENDIX}

Proof of Proposition 2: Assume that $\mathbf{p}^{*}$ is a NE for $\mathcal{G}^{\prime}$ but not for $\mathcal{G}$. Then, there exists a $p_{f}$ such that $\left(p_{f}, \mathbf{p}_{-f}^{*}\right) \in \mathcal{P}$ and

$$
U_{f}\left(\gamma_{f}\left(p_{f}, \mathbf{p}_{-f}^{*}\right)\right)>U_{f}\left(\gamma_{f}\left(\mathbf{p}^{*}\right)\right) .
$$

Since $U_{f}$ is increasing then $\gamma_{f}\left(p_{f}, \mathbf{p}_{-f}^{*}\right)>\gamma_{f}\left(\mathbf{p}^{*}\right)$. But also $V_{f}$ is an increasing function in $\gamma_{f}$ and

$$
V_{f}\left(\gamma_{f}\left(p_{f}, \mathbf{p}_{-f}^{*}\right)\right)>V_{f}\left(\gamma_{f}\left(\mathbf{p}^{*}\right)\right) .
$$

This contradicts the assumption that $\mathbf{p}^{*}$ is a NE for $\mathcal{G}^{\prime}$. Thus, $\mathbf{p}^{*}$ is a NE for both $\mathcal{G}$ and $\mathcal{G}^{\prime}$.

Proof of Proposition 3 Let $\mathbf{p}^{*}$ be an optimal solution to CCPG. First, note that $\mathbf{p}^{*}$ is an NE. If it was not, then there would exist a $p_{f}^{\prime}$ such that $\left(p_{f}^{\prime}, \mathbf{p}_{-f}\right) \in \mathcal{P}, f \in \mathcal{F}$ such that

$$
u_{f}\left(p_{f}^{*}, \mathbf{p}_{-f}^{*}\right)<u_{f}\left(p_{f}^{\prime}, \mathbf{p}_{-f}^{*}\right)
$$

Since $\Phi(\cdot)$ is a potential function, (30) implies that $\Phi\left(p_{f}^{*}, \mathbf{p}_{-f}^{*}\right)<\Phi\left(p_{f}^{\prime}, \mathbf{p}_{-f}^{*}\right)$. This contradicts the fact that $\mathbf{p}^{*}$ is an optimal solution.

Since $\mathbf{p}^{*}$ is an optimal solution, thus, according to the KKT conditions there exists a $\boldsymbol{\nu}=\left(\nu_{1}, \ldots, \nu_{M}\right)^{T} \succeq \mathbf{0}_{M}$ such that

$$
\frac{\partial \Phi}{\partial p_{f}}-\boldsymbol{\nu} \widehat{\mathbf{h}}^{f}=0 \quad \forall f \in \mathcal{F}
$$


at $\mathbf{p}=\mathbf{p}^{*}$, with

$$
\nu_{m}\left(\mathbf{p}^{T} \widehat{\mathbf{h}}_{m}-I_{T}\right)=0 \quad \forall m .
$$

Identifying $\boldsymbol{\lambda}$ in (7)-(9) with $\boldsymbol{\nu}$ we can easily discern that $\mathbf{p}^{*}$ is indeed an NNE. Hence, the result follows.

Proof of Lemma 1 Since $U_{f}\left(\gamma_{f}^{\prime}\right)$ does not depend on $\mathbf{p}_{-f}$, for any $f \in \mathcal{F}$

$$
\begin{aligned}
& \Phi\left(p_{f}, \mathbf{p}_{-f}\right)-\Phi\left(p_{f}^{\prime}, \mathbf{p}_{-f}\right)= \\
& U_{f}\left(\gamma_{f}^{\prime}\left(p_{f}, \mathbf{p}_{-f}\right)\right)-U_{f}\left(\gamma_{f}^{\prime}\left(p_{f}^{\prime}, \mathbf{p}_{-f}\right)\right)
\end{aligned}
$$

for any $\left(p_{f}, \mathbf{p}_{-f}\right),\left(p_{f}^{\prime}, \mathbf{p}_{-f}\right) \in \mathcal{P}$. This proves that $\Phi(\cdot)$ is a potential function.

By the definition of $\Phi(\cdot)$ and $\gamma_{f}^{\prime}$, it is clear that if $U_{f}(\cdot)$ is strictly concave $\forall f \in \mathcal{F}$, then so $\Phi(\cdot)$ is.

Proof of Lemma 2 Note that

$$
\Phi\left(p_{f}, \mathbf{p}_{-f}\right)-\Phi\left(p_{f}^{\prime}, p_{-f}\right)=\log \left(p_{f}\right)-\log \left(p_{f}^{\prime}\right) .
$$

But,

$$
\begin{aligned}
& \log \left(\gamma_{f}\left(p_{f}, \mathbf{p}_{-f}\right)\right)-\log \left(\gamma_{f}\left(p_{f}^{\prime}, p_{-f}\right)\right)= \\
& \log \left(\frac{p_{f} h_{f}}{\mathbf{p}_{-f}^{T} \widetilde{\mathbf{h}}^{f}+\sigma^{2}}\right)-\log \left(\frac{p_{f}^{\prime} h_{f}}{\mathbf{p}_{-f}^{T} \widetilde{\mathbf{h}}^{f}+\sigma^{2}}\right)= \\
& \log \left(p_{f}\right)-\log \left(p_{f}^{\prime}\right) .
\end{aligned}
$$

Thus, comparing (33) and (34) we conclude that $\Phi(\mathbf{p})$ is a potential function. It is easy to verify that $\Phi(\mathbf{p})$ is a strictly concave function in $\mathbf{p}$.

Proof of Lemma 3: Note that

$$
\begin{aligned}
\frac{\partial^{2} U_{f}}{\partial p_{f}^{2}} & =-\frac{B h_{f}^{2}}{\left(p_{f} h_{f}+\mathbf{p}_{-f}^{T} \widetilde{\mathbf{h}}^{f}+\sigma^{2}\right)^{2}} \\
\frac{\partial^{2} U_{f}}{\partial p_{f} \partial p_{\ell}} & =-\frac{B h_{f} \widetilde{h}_{f}^{\ell}}{\left(p_{f} h_{f}+\mathbf{p}_{-f}^{T} \widetilde{\mathbf{h}}+\sigma^{2}\right)^{2}} .
\end{aligned}
$$

Additionally, the maximum of the denominator of $\frac{\partial^{2} U_{f}}{\partial p_{f}^{2}}$ is obtained when $p_{f}, f \in \mathcal{F}$ attains its maximum value in $\mathcal{P}$. Note that for all feasible $p_{f}, f \in \mathcal{F}$ we must have

$$
p_{f} \leq \min _{m} \frac{I_{T}}{\widehat{h}_{m}^{f}}=\bar{p}_{f} .
$$

On the other hand, the minimum value ${ }^{12}$ of the denominator of $\frac{\partial^{2} U_{f}}{\partial p_{f} \partial p_{\ell}}$ and $\frac{\partial^{2} U_{f}}{\partial^{2} p_{f}}$ is $\sigma^{4}$. Since Assumption 1 is satisfied, thus, by identifying $k_{f}$ with $\frac{B h_{f}}{\left(p_{f} h_{f}+\mathbf{p}_{-f}^{T} \widetilde{\mathbf{h}}^{f}+\sigma^{2}\right)^{2}}$ we conclude that the matrix $-\mathbf{G}(\mathbf{p})$ defined in (10) is row-wise and column wise diagonally dominant for all $\mathbf{p} \in \mathcal{P}$. Hence, $-(\mathbf{G}(\mathbf{p})+$ $\left.\mathbf{G}^{T}(\mathbf{p})\right)$ is positive definite $\forall \mathbf{p} \in \mathcal{P}$. Hence, the result follows from Proposition 1.

Proof of Observation 1 Let us assume we have a NNE $\overline{\mathbf{p}}=\left(\bar{p}_{1}, \ldots, \bar{p}_{F}\right)$ such that $\overline{\mathbf{p}}^{T} \widehat{\mathbf{h}}<I_{T}$. Now, consider the following unilateral deviation of player $f$ : It increases its power $\bar{p}_{f}$ to $\tilde{p}_{f}$ such that $\sum_{\ell \neq f} \bar{p}_{\ell} \hat{h}^{\ell}+\tilde{p}_{f} \hat{h}^{f}=I_{T}$, which is a feasible solution. Since $U_{f}\left(\gamma_{f}\right)$ is a strictly increasing

\footnotetext{
${ }^{12}$ The minimum value is obtained when $\mathbf{p}=\mathbf{0}_{F}$.
}

in $p_{f}$ thus, secondary-BS $f$ gets strictly higher utility at $\tilde{p}_{f}$ compared to $\bar{p}_{f}$. Thus, player $f$ has an incentive to deviate unilaterally which, indeed, entails that $\overline{\mathbf{p}}$ cannot be an NE. Hence, the result follows.

Proof of Proposition 6: We use the following result to prove proposition 6. Note that if $i \notin K^{I}$ for some $I$, then $p_{i}=0$, thus from (25) we obtain for any index $k \in K^{I}$

$$
p_{k} h_{k}+\mathbf{p}_{-k}^{T} \widetilde{\mathbf{h}}^{k}=\frac{B h_{k}}{\lambda h^{k}}-\sigma^{2}
$$

Now we are ready to prove the proposition.

If $K^{0}$ is empty, the optimal solution is $\mathbf{p}^{*}=0$. Hence, the proposition is trivially true. Thus, we consider the case when $K^{0}$ is not empty.

Let $L+1$ be the last iteration and thus $\mathbf{p}^{L+1} \geq 0$. Note that for $p_{k}, K \notin K^{L+1}=0$. Thus, in order to conclude the optimality we have to check whether $p_{k}, k \notin K^{L+1}$ satisfies (22)i.e. $p_{k}, k \notin K^{L+1}$ must satisfy the following

$$
\begin{gathered}
\sum_{j \neq k} p_{j}^{L+1} \tilde{h}_{j}^{k} \geq \frac{B h_{k}}{\lambda \hat{h}^{k}}-\sigma^{2} \\
\sum_{j \neq k} p_{j}^{L+1} \frac{\tilde{h}_{j}^{k}}{h_{k}} \geq \frac{B}{\lambda \hat{h}^{k}}-\frac{\sigma^{2}}{h_{k}}
\end{gathered}
$$

First we show that for $k \in K^{L}, \notin K^{L+1}$, the above inequality holds. Later we show the above for $k \mathrm{~s}$ which do not belong to $K^{L}$.

Note that since $k \in K^{L}$ and $p_{i}^{L}=0, i \notin K^{L}$, thus we can write from (35)

$$
p_{k}^{L} h_{k}+\mathbf{p}_{-k}^{T} \widetilde{\mathbf{h}}^{k}=\frac{B h_{k}}{\lambda \hat{h}^{k}}-\sigma^{2}
$$

Since $k \in K^{L}$ and $k \notin K^{L+1}$ thus $p_{k}^{L}<0, p_{k}^{L+1}=0$.

Let $j \in K^{L+1}$. Thus, from (35)

$$
p_{j}^{L+1}+\sum_{i \neq j} p_{i}^{L+1} \frac{\tilde{h}_{i}^{j}}{h_{j}}=\frac{B}{\lambda \hat{h}^{j}}-\frac{\sigma^{2}}{h_{j}}
$$

Since $j \in K^{L+1}$, thus $j \in K^{L}$. Thus, from (35)

$$
p_{j}^{L}+\sum_{i \neq j} p_{i}^{L} \frac{\tilde{h}_{i}^{j}}{h_{j}}=\frac{B}{\lambda \hat{h}^{j}}-\frac{\sigma^{2}}{h_{j}}
$$

Thus, subtracting (39) from (38) we obtain

$$
p_{j}^{L+1}+\sum_{i \neq j} p_{i}^{L+1} \frac{\tilde{h}_{i}^{j}}{h_{j}}-p_{j}^{L}-\sum_{i \neq j} p_{i}^{L} \frac{\tilde{h}_{i}^{j}}{h_{j}}=0
$$

Note that the above expression is true for any $j \in K^{L+1}$. Let $P_{1}=\left\{i: p_{i}^{L+1} \geq p_{i}^{L}\right\}$ and $P_{2}=P_{1}^{C}$. Since $p_{k}^{L+1}>p_{k}^{L}$ (as $p_{k}^{L+1}=0, p_{k}^{L}<0$ ), thus $P_{1}$ is not empty. From (40) we obtain

$$
p_{j}^{L+1}-p_{j}^{L} \geq \sum_{i \in P_{1}, \neq j}\left(p_{i}^{L}-p_{i}^{L+1}\right) \frac{\tilde{h}_{i}^{j}}{h_{j}}
$$

If $i \notin K^{L+1}$, then $p_{i}^{L+1}=0$ and $p_{i}^{L} \leq 0$, thus by the definition of $P_{2}$, all indices $j \in P_{2}$ must belong to $K^{L+1}$. Thus, (41) is 
valid for any $j \in P_{2}$. Thus,

$$
\begin{aligned}
& \sum_{j \in P_{2}}\left(p_{j}^{L+1}-p_{j}^{L}\right) \frac{\tilde{h}_{j}^{k}}{h_{k}}+\sum_{i \in P_{1} /\{k\}}\left(p_{i}^{L+1}-p_{i}^{L}\right) \frac{\tilde{h}_{i}^{k}}{h_{k}}+p_{k}^{L+1}-p_{k}^{L} \\
& \geq \sum_{j \in P_{2}}\left(\sum_{i \in P_{1}}\left(p_{i}^{L}-p_{i}^{L+1}\right) \frac{\tilde{h}_{i}^{j} \tilde{h}_{j}^{k}}{h_{k} h_{j}}\right) \\
& +\sum_{i \in P_{1} /\{k\}}\left(p_{i}^{L+1}-p_{i}^{L}\right) \frac{\tilde{h}_{i}^{k}}{h_{k}}+p_{k}^{L+1}-p_{k}^{L} \quad(\text { from (41)) } \\
& =\left(p_{k}^{L+1}-p_{k}^{L}\right)\left(1-\sum_{j \in P_{2}} \frac{\tilde{h}_{k}^{j} \tilde{h}_{j}^{k}}{h_{k} h_{j}}\right) \\
& +\sum_{i \in P_{1} /\{k\}}\left(p_{i}^{L+1}-p_{i}^{L}\right)\left(-\sum_{j \in P_{2}} \frac{\tilde{h}_{j}^{k} \tilde{h}_{i}^{j}}{h_{k} h_{j}}+\frac{\tilde{h}_{i}^{k}}{h_{k}}\right)
\end{aligned}
$$

Since $p_{i}^{L+1}-p_{i}^{L} \geq 0 \forall i \in P_{1}$, thus, from assumption 3 and (42) we have

$$
\begin{aligned}
& \sum_{j \in P_{2}} p_{j}^{L+1} \frac{\tilde{h}_{j}^{k}}{h_{k}}+\sum_{j \in P_{1} /\{k\}} p_{j}^{L+1} \frac{\tilde{h}_{j}^{k}}{h_{k}}+p_{k}^{L+1} \\
& \geq p_{k}^{L}+\sum_{j \neq k} p_{j}^{L} \frac{\tilde{h}_{j}^{k}}{h_{k}} \\
& =\frac{B}{\lambda \hat{h}^{k}}-\frac{\sigma^{2}}{h_{k}} \quad(\text { from }(37))
\end{aligned}
$$

Since $p_{k}^{L+1}=0$,hence from (43) we obtain (36) is valid for all $k \in K^{L}$ but not in $K^{L+1}$.

Now, suppose that $k \in K^{L-1}$ but $k \notin K^{L}$. Thus, $p_{k}^{L}=$ $p_{k}^{L+1}=0$. Since $k \in K^{L-1}$, thus from (35)

$$
p_{k}^{L-1} h_{k}+\sum_{j \neq k} p_{j}^{L-1} \tilde{h}_{j}^{k}=\frac{B h_{k}}{\lambda \hat{h}^{k}}-\sigma^{2}
$$

Suppose $j \in K^{L+1}$. Then, from (35)

$$
p_{j}^{L+1} h_{j}+\sum_{i \neq j} p_{i}^{L+1} \tilde{h}_{i}^{j}=\frac{B h_{j}}{\lambda \hat{h}^{j}}-\sigma^{2}
$$

Since $j \in K^{L+1}$, thus $j \in K^{L-1}$. Hence,

$$
p_{j}^{L-1} h_{j}+\sum_{i \neq j} p_{i}^{L-1} \tilde{h}_{i}^{j}=\frac{B h_{j}}{\lambda \hat{h}^{j}}-\sigma^{2}
$$

Thus, from (45) and (46) we obtain

$$
\begin{aligned}
& p_{j}^{L+1}-p_{j}^{L-1}+\sum_{i \neq j}\left(p_{i}^{L+1}-p_{i}^{L-1}\right) \frac{\tilde{h}_{i}^{j}}{h_{j}}=0 \\
& p_{j}^{L+1}-p_{j}^{L-1} \geq \sum_{i \in K_{1}, \neq j}\left(p_{i}^{L-1}-p_{i}^{L+1}\right) \frac{\tilde{h}_{i}^{j}}{h_{j}}
\end{aligned}
$$

where $K_{1}=\left\{i: p_{i}^{L+1} \geq p_{i}^{L-1}\right\}$; let $K_{2}=K_{1}^{C}$. Since $k \in$ $K^{L-1}$ but $k \notin K^{L}$, thus $p_{k}^{L-1}<0=p_{k}^{L+1}$. Thus, $K_{1}$ is not empty. Now, suppose that $i \in K^{L}$, but $i \notin K^{L+1}$, we have already shown that (36) is valid for those indices, thus from (36) we have

$$
p_{i}^{L+1}+\sum_{l \neq i} p_{l}^{L+1} \frac{\tilde{h}_{l}^{i}}{h_{i}} \geq \frac{B}{\lambda \hat{h}^{i}}-\frac{\sigma^{2}}{h_{i}}
$$

where $p_{i}^{L+1}=0$.

Again since $i \in K^{L}$, thus, $i \in K^{L-1}$. Thus from (35), we obtain

$$
\begin{aligned}
& p_{i}^{L-1} h_{i}+\sum_{l \neq i} p_{l}^{L-1} \tilde{h}_{l}^{i}=\frac{B h_{i}}{\lambda \hat{h}^{i}}-\sigma^{2} \\
& p_{i}^{L+1}-p_{i}^{L-1}+\sum_{l \neq i}\left(p_{l}^{L+1}-p_{l}^{L-1}\right) \frac{\tilde{h}_{l}^{i}}{h_{i}} \geq 0 \\
& p_{i}^{L+1}-p_{i}^{L-1} \geq \sum_{l \in K_{1}, \neq i}\left(p_{l}^{L-1}-p_{l}^{L+1}\right) \frac{\tilde{h}_{l}^{i}}{h_{i}}
\end{aligned}
$$

Note that if $a \notin K^{L}$, then $a \notin K^{L+1}$. Thus, $p_{a}^{L+1}=0$ and $p_{a}^{L-1} \leq 0$. Thus, an index $i \in K_{2}$ only if $i \in K^{L}$ or $i \in K^{L+1}$. Thus, (49) is valid for any index $i \in K^{2}$. Hence

$$
\begin{aligned}
& \sum_{j \in K_{2}}\left(p_{j}^{L+1}-p_{j}^{L-1}\right) \frac{\tilde{h}_{j}^{k}}{h_{k}} \\
& +\sum_{j \in K_{1}, \neq k}\left(p_{j}^{L+1}-p_{j}^{L-1}\right) \frac{\tilde{h}_{j}^{k}}{h_{k}}+p_{k}^{L+1}-p_{k}^{L-1} \\
& \geq \sum_{j \in K_{2}}\left(\sum_{i \in K_{1}}\left(p_{i}^{L-1}-p_{i}^{L+1}\right) \frac{\tilde{h}_{i}^{j} \tilde{h}_{j}^{k}}{h_{j} h_{k}}\right) \\
& +\sum_{i \in K_{1}, i \neq k}\left(p_{i}^{L+1}-p_{i}^{L-1}\right) \frac{\tilde{h}_{i}^{k}}{h_{k}}+p_{k}^{L+1}-p_{k}^{L-1} \\
& =\sum_{i \in K_{1} /\{k\}}\left(p_{i}^{L+1}-p_{i}^{L-1}\right)\left(\frac{\tilde{h}_{i}^{k}}{h_{k}}-\sum_{j \in K_{2}} \frac{\tilde{h}_{i}^{j} \tilde{h}_{j}^{k}}{h_{j} h_{k}}\right) \\
& +\left(p_{k}^{L+1}-p_{k}^{L-1}\right)\left(1-\sum_{j \in K_{2}} \frac{\tilde{h}_{i}^{j} \tilde{h}_{j}^{k}}{h_{j} h_{k}}\right)
\end{aligned}
$$

Since $p_{i}^{L+1}-p_{i}^{L-1} \geq 0$ for $i \in K_{1}$, thus, from assumption 3 and (50) we have

$$
\begin{aligned}
& \sum_{j \in K_{2}} p_{j}^{L+1} \frac{\tilde{h}_{j}^{k}}{h_{k}}+\sum_{j \in K_{1} /\{k\}} p_{j}^{L+1} \frac{\tilde{h}_{j}^{k}}{h_{k}}+p_{k}^{L+1} \\
& \geq p_{k}^{L-1}+\sum_{j \in K_{2}} p_{j}^{L-1} \frac{\tilde{h}_{j}^{k}}{h_{k}}+\sum_{j \in K_{1} /\{k\}} p_{j}^{L-1} \frac{\tilde{h}_{j}^{k}}{h_{k}} \\
& =\frac{B}{\lambda \hat{h}_{k}}-\sigma^{2} \quad(\text { from }(44))
\end{aligned}
$$

Since $p_{k}^{L+1}=0$, thus, (51) implies that (36) is valid for $k \in$ $K^{L-1}$, but $\notin K^{L+1}, K^{L}$.

Note that we have only used the fact that (36) is valid for any $i \in K^{L}$ but $i \notin K^{L+1}$ in order to show that (36) is valid for a $k \in K^{L-1}$ but $k \notin K^{L}, K^{L+1}$. Hence, using the same argument we can show that (36) is valid for $k \in K^{I}$ but $k \notin K^{I+1}$ for any $I<L-1$. Hence, the result follows. 